\title{
A Simplified Approach for the Rapid Generation of Transient Heat-Shield Environments
}

\author{
Kathryn E. Wurster ${ }^{*}$ and E. Vincent Zoby ${ }^{\dagger}$ \\ NASA Langley Research Center, Hampton, VA 23681 \\ Janelle C. Mills* \\ Vigyan, Inc., Hampton, VA, 23666 \\ and \\ Hilmi Kamhawi ${ }^{\S}$ \\ TechnoSoft, Inc., Cincinnati, Ohio 45242
}

\begin{abstract}
A simplified approach has been developed whereby transient entry heating environments are reliably predicted based upon a limited set of benchmark radiative and convective solutions. Heating, pressure and shear-stress levels, non-dimensionalized by an appropriate parameter at each benchmark condition are applied throughout the entry profile. This approach was shown to be valid based on the observation that the fully catalytic, laminar distributions examined were relatively insensitive to altitude as well as velocity throughout the regime of significant heating. In order to establish a "best prediction" by which to judge the results that can be obtained using a very limited benchmark set, predictions based on a series of benchmark cases along a trajectory are used. Solutions which rely only on the limited benchmark set, ideally in the neighborhood of peak heating, are compared against the resultant transient heating rates and total heat loads from the "best prediction." Predictions based on using two or fewer benchmark cases at or near the trajectory peak heating condition, yielded results to within 5-10 percent of the "best predictions." Thus, the method provides transient heating environments over the heat-shield face with sufficient resolution and accuracy for thermal protection system design and also offers a significant capability to perform rapid trade studies such as the effect of different trajectories, atmospheres, or trim angle of attack, on convective and radiative heating rates and loads, pressure, and shear-stress levels.
\end{abstract}

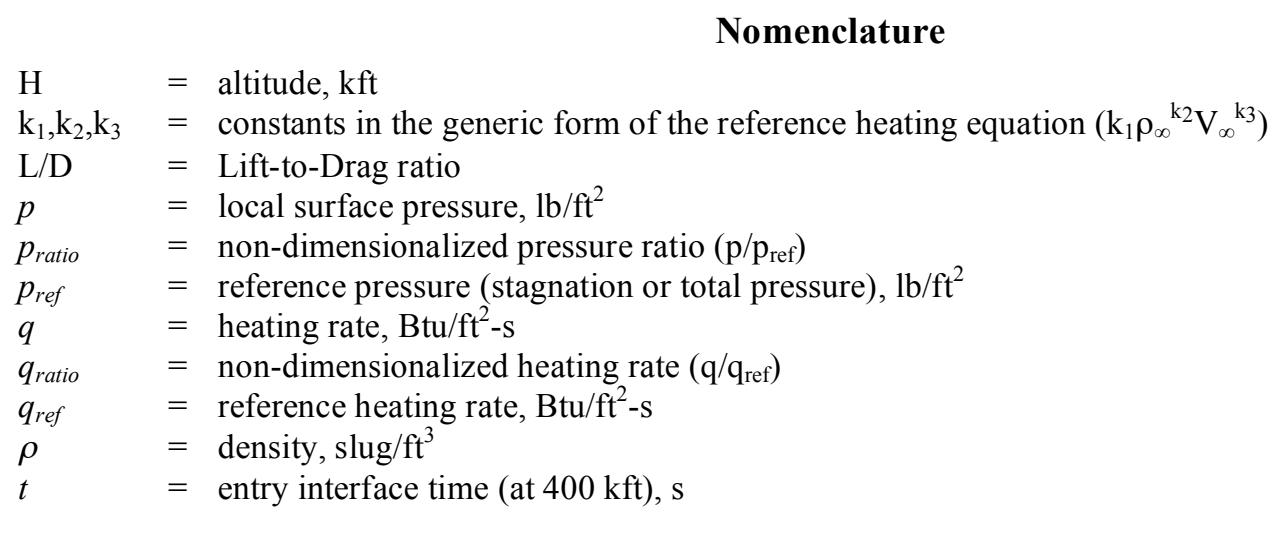

\footnotetext{
* Senior Research Engineer, Vehicle Analysis Branch, Mail Stop 451, Associate Fellow AIAA.

${ }^{\dagger}$ Senior Research Engineer, Aerothermodynamics Branch, Mail Stop 408A, Fellow AIAA.

* Computer Scientist, Mail Stop 451.

$\S$ Software Engineer, Mail Stop 451.
} 
$\begin{array}{ll}\mathrm{T} & =\text { temperature, } \operatorname{deg} \mathrm{R} \\ V & =\text { velocity, } \mathrm{kft} / \mathrm{s}\end{array}$

Subscripts

c, conv $=$ convective

$r$ rad $=$ radiative

$\infty \quad=$ freestream

\section{Introduction}

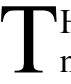
HE National Aeronautics and Space Administration (NASA) is formulating a program to return humans to the moon by the end of the next decade, as set forth in the president's Vision for Space Exploration introduced in 2004. Since the early 1980's NASA's focus has been on reusable vehicles and thermal protection systems (TPS) for return at the relatively low-velocity conditions associated with low-earth-orbit (LEO) missions such as the International Space Station (ISS) and the Hubble telescope servicing missions. Aeroheating environments for lunar return missions at $\sim 35 \mathrm{kft} / \mathrm{s}$ will be significantly higher when compared to LEO return at $\sim 25 \mathrm{kft} / \mathrm{s}$. Reusable TPS such as those employed on the Shuttle orbiter cannot withstand the nominal environments associated with lunar return missions. The proposed lunar return concept, the Crew Exploration Vehicle (CEV), is a low lift/drag vehicle similar to the Apollo Command Module (CM) and will employ an ablative heat-shield concept like the Apollo. A drawing of the CEV entry module is illustrated in Figure 1 showing the dimensions in feet. The vehicle is approximately $28 \%$ larger scale than the Apollo CM and has a base heat-shield diameter of $16.5 \mathrm{ft}(5 \mathrm{~m})$ - compared to $12.83 \mathrm{ft}(3.912 \mathrm{~m})$ for the Apollo CM. Critical to the design of the heat shield are the transient aeroheating environments against which the crew and structure must be protected. Primary responsibility for determination of those environments lies with NASA. The methodology described here is intended to complement the approach selected by the CEV Aerosciences Project (CAP). The CAP approach relies upon the population of an aerothermal database with a limited number of computational solutions for convective and radiative heating, and "densification" of the trajectory space using an engineering method $^{1}$ anchored to the database cases. NASA will provide the database and the tools to interpolate the database, defined in Mach, dynamic pressure, angle-of-attack (AOA) space, for the CEV geometry for any given entry trajectory. While the CAP will provide full-body aerothermal environments, the approach discussed herein focuses on the aeroheating environments critical to the design of the primary forebody heat shield only. One advantage of the proposed methodology is that no large database is required in order to generate the environments for an entry

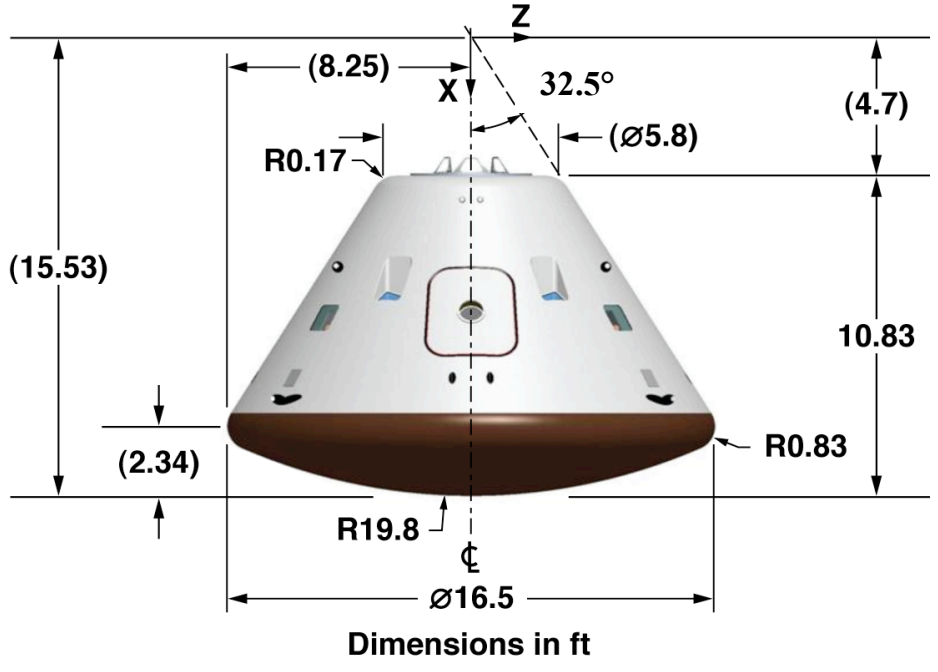

Figure 1. Crew Exploration Vehicle. trajectory.

Thus, the primary objective of the present effort is to provide a quick, but reliable independent assessment capability for the program in order to reduce risk. A secondary objective is to enable rapid performance of required trade studies, such as evaluation of TPS requirements for evolving mission profiles, including the impact of atmospheric and trajectory dispersions, changes in trim AOA, weight growth, and changing mission requirements. The implication of the uncertainty levels on TPS design can also be examined using the proposed approach. Additionally, this rapid prediction method can be utilized to establish materials testing requirements, evaluate the suitability of existing test facilities and to support the need for new facilities and/or flight testing. 


\section{Background}

In 2004, NASA responded to the president's Vision for Space Exploration first, by initiating a number of Exploration Systems Research and Technology programs as part of the Constellation program and then, by bringing together, in May of 2005, a team of experts from around the agency to perform an Exploration Systems Architecture Study $^{2}$ (ESAS). This study was conducted within a 90-day time period during the spring/summer of 2005. While much was accomplished, ESAS was primarily an architecture study with little attention to detailed vehicle design. Subsequently, NASA Administrator Dr. Michael Griffin, tasked what he called a "Smart Buyer Design Team" ${ }^{\text {" to }}$ "flesh out" more of the vehicle design issues for the CEV. This study was also an extremely fast paced-effort with a 90-day timeline (Jan-Mar 2006) from initiation to final report, during which a team of nearly 300 experts from the agency worked to develop an integrated vehicle design and to consider several promising design alternatives beyond those envisioned by the original ESAS team. A secondary goal was to re-educate NASA in the vehicle design issues associated with the manned return of a low L/D vehicle from the moon, a scenario rarely considered in the decades following the end of the Apollo program in 1974. A particular area of concern was the ablative heat-shield design. While ablators continue to be used in planetary entry applications, manned Earth entry using an ablative heat shield was a technology area that had received little or no attention over recent years. The Smart Buyer Team was fortunate to have the participation of Dr. Donald Curry who was a primary contributor to the design of the original Apollo heat shield. ${ }^{4}$ In order for the team to perform a preliminary heat-shield design and to trade the various heat-shield materials proposed for the broad range of entry profiles and conditions under consideration, it was necessary to develop an approach whereby transient heat-shield heating and pressure environments could be rapidly and accurately generated. Required trades included Lunar/LEO/Mars return scenarios, laminar/turbulent environments, and uncertainties in radiative heating levels, particularly for the high-velocity Mars return cases. At that time, the CAP had produced only a limited set of benchmark solutions, the majority of which were for the original $18.04 \mathrm{ft}$ $(5.5 \mathrm{~m})$ diameter vehicle and was not prepared to provide the environments in the time frame required by the Smart Buyer TPS design team. However, since the intent of the Smart Buyer Team was to conduct their study as independently as possible from the CAP team effort, the approach described here was successfully formulated, implemented, validated and reported within the three-month scope of the Smart Buyer activity. Ongoing development has further extended the capability and allowed for additional validation of the procedure. The procedure has been adopted by the CAP team as secondary approach to 1) perform quick-look trade studies to assess various design issues, 2) evaluate the impact of uncertainties on the heat-shield design, and 3) provide verification checks for the baseline procedure.

\section{Methodology}

\section{A. Approach/Assumptions}

The methodology developed relies entirely upon the accuracy of a limited set of available benchmark solutions within the trajectory space, together with the judicious selection of the cases used to model the heating for a given trajectory. The present approach does not predict the heating in the absence of any benchmark computational solutions, but rather provides a technique by which a limited set of benchmarks can be used to derive transient heating environments over the entire entry profile to a level of accuracy and detail sufficient to perform heat-shield design trades. The basic premise of the present approach relies on the assumption that the heating, pressure, and shear-stress distributions over the face of the heat-shield can be non-dimensionalized in such a fashion that they remain relatively constant for the duration of the main heating pulse. However, the approach does not require that the benchmark cases lie directly on any given trajectory. The relatively small cg envelope for the CEV and the resultant limitations on the AOA at which the vehicle trims contributes to the validity of this assumption (based on the available benchmark heating data). For all trajectories considered, the AOA throughout the primary heat pulse remains at the nominal trim angle, plus or minus $\sim 1.5^{\circ}$. Initial designs were flown with the Lunar/LEO returns generally at or about $27^{\circ}\left(153^{\circ}\right.$ in the aero coordinate system). Packaging constraints appear to be driving the trim AOA down due to limited cg offset capability, but the nominal, plus or minus $\sim 1.5^{\circ}$, remains a valid assumption over the duration of the primary heat pulse.

Solutions based on two Navier-Stokes codes, LAURA ${ }^{5}$ and DPLR $^{6}$ (Data-Parallel Lower-Upper Relaxation Method) provide the laminar and turbulent convective heating benchmark calculations for population of the CAP aerothermal database. ${ }^{7}$ Note that for this study only the laminar benchmark results are employed. All solutions in the database assume a fully catalytic wall boundary condition and an emissivity of 0.85 is used to compute the radiation equilibrium wall temperature and provide the hot wall condition on which the heating rates are based. At present, 
radiative solutions generated using NEQAIR ${ }^{8}$ are provided only for conditions at velocities of $\sim 28 \mathrm{kft} / \mathrm{s}$ or greater. Coupling effects between the convective flowfield and the radiation are not considered in the database solutions.

A non-dimensionalization parameter that can be expected to track the level of heating throughout the trajectory profiles was required in order to determine the time-dependent heating levels. The present approach relies on a hotwall Fay Riddell ${ }^{9}$ stagnation heating value, as predicted using MINIVER, ${ }^{10}$ to non-dimensionalize the convective heating distributions. A simple function of the form $\mathrm{k}_{1} \rho_{\infty}{ }^{{ }^{2} 2} \mathrm{~V}_{\infty}{ }^{\mathrm{k} 3}$ could be explored as a reference parameter (where $\mathrm{k}_{2}$ $=1$ and $\mathrm{k}_{3}=3$ provide the correct dimensional form), as could a number of stagnation heating predictions methods including Hoshizaki, ${ }^{11}$ Cohen, ${ }^{12}$ Sutton and Graves, ${ }^{13}$ etc. The fundamental requirement for the nondimensionalizing or reference value is that it be predicated on parameters easily obtained from the freestream conditions and that it reliably predict the nature of the heating as a function of time within the trajectory space. The reference hot wall value for non-dimensionalization, qref $_{\text {conv }}$, is computed at the same freestream conditions $\left(\mathrm{p}_{\infty}, \mathrm{T}_{\infty}\right.$, $\rho_{\infty}, V_{\infty}$ ) and emissivity used for the original benchmark case. The time-dependent reference value computed as a function of the trajectory conditions allows prediction of the environments away from the benchmark cases. The non-dimensionalized distribution is simply multiplied by the reference parameter computed at each point along the trajectory to obtain the dimensional heating rates, pressures and shear stresses.

The benchmark radiative heating solutions are non-dimensionalized by a reference value derived from the work of Sutton and Hartung ${ }^{14}$ and Tauber. ${ }^{15}$ Considerable effort has been expended by the CAP team in order to obtain a more reliable prediction of radiative heating levels. The effort has been hampered by the lack of relevant flight data and the limited resources devoted to the technology area in the intervening years since the design of the Apollo spacecraft. As part of the CAP team effort, benchmark radiative heating estimates based on RADEQUIL ${ }^{16}$ solutions for lower velocities and altitudes than those heretofore considered were provided for this study. When the heating was determined to be significant beyond the density, velocity range in Ref. 14 for the assumed CEV radius, the original tables were extended to support the development of the present approach and to compute the radiative reference values. Once again, the reference value is computed along the trajectory and combined with the nondimensionalized distribution to determine dimensional radiative heating rates. The convective and radiative rates are simply added together to obtain the total. Typical workhorse tools for ablative heat-shield analysis, $\mathrm{CMA}^{17}$ and FIAT ${ }^{18}$ import the uncoupled rates and perform the thermochemistry analysis based upon the air/heat-shield material interactions to account for surface decomposition and recession. Flowfield coupling effects were not taken into account.

In order to generate the reference convective and radiative heating rates, an effective radius is selected based on the current range of trim AOA. Utilization of an effective radius approach as a function of AOA is under consideration as a potential solution to account for large AOA variations, if warranted, based on any future vehicle design changes or for extension of the present approach to vehicles with less restrictive trim limits.

A similar non-dimensionalization procedure is performed to enable prediction of the surface pressure and shearstress distributions. The benchmark cases are simply non-dimensionalized by the freestream total pressure. Because the pressure distributions over the critical range of conditions (aeroheating) are essentially Newtonian on the forward face of the heat-shield, time will not be devoted here explicitly to validating the non-dimensionalization approach for pressure distributions. The pressure distributions are however presented along with the heating distributions so the reader can make the assessment.

\section{B. Validation}

Validation of the proposed engineering technique was conducted in three phases. First it was necessary to establish that the underlying assumptions of the approach are valid for the desired vehicle trajectory design space. Figures 2 and 3 illustrate the altitude-velocity $(\mathrm{H}-\mathrm{V})$ and AOA-velocity space respectively for the range of entry modes and profiles currently under consideration for the CEV. Included are entry profiles designated here as $28 \mathrm{deg}$ and 20 deg, roughly bounding the range of the trim AOA expected for the CEV. Despite the relatively large range in the prescribed trim AOA, the altitude-velocity flight profiles remain within a relatively narrow band for each respective entry mode - lunar ballistic, lunar skip (nominal), and LEO (ISS) ballistic and guided return. Note, the lunar guided return is ignored in this discussion as there is no comparable trajectory available for the 20-deg trim case. The period of high heating on the lunar returns extends from roughly $34.5-28 \mathrm{kft} / \mathrm{s}$, and that for the LEO returns from approximately 24-19.5 kft/s. As illustrated in Fig. 2, the maximum difference in altitude for a given velocity condition (over the period of high heating) for the two trim AOA is $\sim 17 \mathrm{kft}$ (@ V $234.5 \mathrm{ft} / \mathrm{s}$ ) for the lunar returns, and $\sim 22 \mathrm{kft}$ (@ V 19.5 kft/s) for nominal LEO return (guided). In the case of the LEO ballistic return, the altitudes are virtually identical for both trim-AOA profiles. In both the lunar and the LEO return cases, the separation in altitude for the primary heating pulseis $\sim 5 \mathrm{kft}$. Although there is a large difference in the two trim AOA conditions currently being considered, the trim AOA for each of the entry trajectories shown in Fig. 2 also 
remains within a narrow band (Fig. 3) during the likely high-heating period identified previously. It is clear from Fig. 3 that the trajectory designated " 28 " actually trims at about 26.5 deg over the expected period of peak heating. The 28 deg designation is a legacy from the ESAS where an L/D significantly higher than that for Apollo was assumed in order to meet landing requirements. The oscillation apparent only in the 20-deg case is typical of a 6-dof (degree of freedom) simulation including winds, etc. The "smooth" profile for the " 28 " degree entry is more typical of a 3-dof simulation.

Having established the first assumption on which this approach is based, i.e. the narrow H-V space, constant AOA over the critical heating regime, the second phase of the validation effort explored the assumption that nondimensionalized heating and pressure distributions for a given AOA are relatively insensitive to both altitude and velocity variation in the critical region near peak heating. Symmetry plane cuts are shown throughout the remainder of the paper for clarity and generally reflect the relative degree of agreement over the face of the heat shield. The symmetry plane is a plane defined by the axis of rotation of the Crew Module and a perpendicular extending from the "hot" corner, facing into the flow on entry, to the cool corner oriented away from the flow. The data are shown along the surface of the-heat shield face where the plane of symmetry intersects the surface. In the figures presented throughout the paper, the "hot" corner will appear to the left of the figures (negative z). Limited benchmark data evaluated in the earlier study for the $18.04 \mathrm{ft}$ diameter CEV concept suggested the relative insensitivity of the heating distributions on the heat-shield face to both velocity and angle of attack.

In the approximately nine months since the conclusion of the Smart Buyer activity, the CAP team has populated the Aerothermal Database (ATDB) with many benchmark solutions for the $16.5 \mathrm{ft}$ base diameter CEV. Figures 4

Table 1. Aerothermal Database Case Designation

\section{(a) Velocity}

\begin{tabular}{ccc}
\hline \hline Case Designation & $\begin{array}{c}\text { Condition (English) } \\
\text { Velocity, kft/sec }\end{array}$ & $\begin{array}{c}\text { Condition (metric) } \\
\text { Velocity, km/sec }\end{array}$ \\
\hline V10.15 & 33.300 & 10.15 \\
V9 & 29.528 & 9.00 \\
V8.65 & 28.379 & 8.65 \\
V8 & 26.247 & 8.00 \\
V7 & 22.966 & 7.00 \\
V6 & 19.685 & 6.00 \\
V5 & 16.404 & 5.00 \\
V4 & 13.123 & 4.00 \\
\hline
\end{tabular}

\section{(b) Altitude}

\begin{tabular}{ccc}
\hline \hline Case Designation & $\begin{array}{c}\text { Condition (English) } \\
\text { Altitude, } \mathrm{kft}\end{array}$ & $\begin{array}{c}\text { Condition (metric) } \\
\text { Altitude, } \mathrm{km}\end{array}$ \\
\hline H-70 & 229.659 & 70.00 \\
H-65 & 213.255 & 65.00 \\
H-60.52 & 198.556 & 60.52 \\
H-59 & 193.570 & 59.00 \\
H-52.23 & 171.358 & 52.23 \\
H-48.3 & 158.465 & 48.30 \\
H-48 & 157.480 & 48.00 \\
H-44.5 & 145.997 & 44.50 \\
H-44.0 & 144.357 & 44.00 \\
H-42 & 137.795 & 42.00 \\
H-39.7 & 130.249 & 39.70 \\
H-37.2 & 122.047 & 37.20 \\
\hline \hline
\end{tabular}


and 5 illustrate the array of benchmark computational solutions presently available for the $16.5 \mathrm{ft}$ diameter vehicle. The CAP team used metric designations for all benchmark conditions. Tables $1 \mathrm{a}$ and $1 \mathrm{~b}$ give the designations and the equivalent English conditions, for velocity and altitude respectively. Convective solutions (either LAURA or DPLR) are available for all benchmark conditions shown. NEQAIR radiative heating solutions are presently available only for velocities $>\sim 28 \mathrm{kft} / \mathrm{s}$. In order to assess the altitude sensitivity of the distributions for the 20-deg trim AOA case, four velocity conditions at which solutions were available for two unique altitudes were examined. Figures 6a-6c illustrates the relative insensitivity of the convective heating distributions to altitude variation at 20deg AOA for three of the velocity conditions considered, 16.4, 19.7, and $29.5 \mathrm{kft} / \mathrm{s}(5,6$ and $9 \mathrm{~km} / \mathrm{s})$ respectively. Note that for simplicity in providing the titles and legends for most of the remaining figures, the metric value is shown for the figure. The reader is referred to Tables $1 \mathrm{a}$ and $1 \mathrm{~b}$ for the English equivalent. As stated earlier, it is assumed that the CFD (Computational Fluid Dynamics) benchmark cases are accurate. The reason for the inflection in the solid curve on Fig. 6b (also seen as a "bump" between $z=-4$ and -7 ) however, is unknown at this time. It is apparent, based on Fig. 4, that the altitudes for these computational solutions bound the anticipated trajectory space quite well. Differences in convective heating ratios over the heat-shield face due to altitude variation are observed to range from a maximum of $\sim 5 \%$ at the $29.5 \mathrm{kft} / \mathrm{s}(9 \mathrm{~km} / \mathrm{s})$ condition down to about $2 \%$ for the $16.4 \mathrm{kft} / \mathrm{s}(5 \mathrm{~km} / \mathrm{s})$ case. In each case, the level of the distribution is slightly higher at the higher density/lower altitude condition. Thus, for the present method, if a CFD solution is available for the required AOA and velocity, it can be applied over a large range in altitude with no more than a 5\% loss in accuracy as compared to a detailed computational analysis.

The relative insensitivity of the convective distributions to the velocity conditions is also key to the success of the present technique. In order to ensure that the maximum rates are captured, the technique requires that the engineer have access to a single benchmark solution at or near peak heating. Typically, the expected peak heating condition is the first condition at which a CFD solution is generated. Heat-shield design is dependent not only on peak rate, but also integrated heat load. Therefore, it must be demonstrated that selection of a peak heating condition and application throughout the trajectory profile would not lead to a substantial over-prediction of the heating loads. The nature of the CEV is such that the loads are dominated by a relatively short heat pulse and, therefore, overprediction of the relatively low heating rates at velocities $<10 \mathrm{kft} / \mathrm{s}$, occurring later in the trajectory would not cause large errors in the heat-load prediction. Figure 7 illustrates the velocity sensitivity of the convective heating distribution for the 20-deg AOA, low-altitude cases considered previously in Fig. 6. The inflection noted for the 19.7 $\mathrm{kft} / \mathrm{s}, 146 \mathrm{kft}(6 \mathrm{~km} / \mathrm{s}, 44.5 \mathrm{~km})$ results shown in Fig. 7 is the same one previously identified in Fig. $6 \mathrm{~b}$. The 29.5 $\mathrm{kft} / \mathrm{s}(9 \mathrm{~km} / \mathrm{s})$ case over predicts the $19.7 \mathrm{kft} / \mathrm{s}(6 \mathrm{~km} / \mathrm{s})$ case at the corner by only $2 \%$. Comparing the $29.5 \mathrm{kft} / \mathrm{s}(9$ $\mathrm{km} / \mathrm{s})$ to the well-behaved $16.4 \mathrm{kft} / \mathrm{s}(5 \mathrm{~km} / \mathrm{s})$ case yields an over prediction on the order of only $10 \%$ at the corner and less over the acreage. Based on the limited data set available at this time it appears that, although the distributions are slightly velocity dependent in this range, an over-prediction of less than $10 \%$ would be anticipated for the heating at lower velocities occurring well after the primary heat pulse.

Although the AOA history for the high heating phase of any of the current CEV entry trajectories does not vary significantly, it is important to consider the potential AOA effect if the present approach is to be extrapolated to a similarly shaped vehicle with a larger trim AOA range. Figure 8a illustrates the impact on the symmetry plane heating profile associated with an angle-of-attack variation from 27.5 to 20 degrees. No attempt has been made to account for a change in the effective radius due to the AOA variation. For a large variation in AOA during the trajectory, a significant effect on the distribution (15-20\%) may be encountered. The overall impact, given the same trajectory profile, would likely be an increase in the integrated heating over the acreage of the heat shield with a commensurate reduction on the hot corner (for a reduction in AOA from 27.5 to 20). Employing a variable effective radius may mitigate the differences. In a later section of the paper, the sensitivity of the integrated heat load to AOA is discussed. Figure $8 \mathrm{~b}$ also shows the change in pressure distribution over the same AOA range as Fig $8 \mathrm{a}$.

In the case of radiative heating, the limited solution set precludes any detailed validation of the proposed approach at this time. However, it is important to note two relevant observations. First, the character of the distributions changes as the velocity is decreased from 33.3 to $29.5 \mathrm{kft} / \mathrm{s}(10.15$ to $9 \mathrm{~km} / \mathrm{s})$ and on down to $28.4 \mathrm{kft} / \mathrm{s}$ $(8.65 \mathrm{~km} / \mathrm{s})$, and the trend is illustrated in Fig. 9. The reader is once again referred to tables $1 \mathrm{a}$ and $1 \mathrm{~b}$ for clarification of the line legends. As the velocity decreases, the profile "rounds" out and appears to be very similar at 29.5 and $28.4 \mathrm{kft} / \mathrm{s}$ (9 and $8.65 \mathrm{~km} / \mathrm{s})$. A similar trend was observed in the Smart Buyer study, where an additional benchmark case was available at $\sim 14 \mathrm{~km} / \mathrm{s}$. Note that, despite the variation in altitude (178 kft plus or minus $20 \mathrm{kft}$ ), for the cases shown in Fig. 9, the behavior appears to be consistent with the velocity variation. Figures 10a and 10b illustrate the variation with AOA for a velocity of $29.5 \mathrm{kft} / \mathrm{s}(9 \mathrm{~km} / \mathrm{s})$ first without and then with an effective radius correction. Here the effective radius is designed to account for the variation in shock-layer thickness with angle of attack. The data in Fig. 10a collapse to the data in Fig. 10b when the effective radius correction is performed. The radius chosen was based upon Ried's Apollo work ${ }^{19}$ scaled to the size of the CEV. Figure 11, from Ref. 19, 
illustrates the radiative effective radius for Apollo as a function of AOA. With the correction, the maximum radiation level is observed to be $\sim 80 \%$ of the stagnation value as predicted using Sutton's extended air radiation lookup table. The use of the effective radius concept is proposed as one procedure to extend the technique to handle a time-variant AOA profile. The present technique assumes a constant AOA profile based on the current trajectories, and incorporates any time-dependence through a technique where the distributions are interpolated between benchmarks calculated for different AOA.

In the third phase of the validation, the technique is demonstrated by comparing the prediction based on a single benchmark solution (one convective + one radiative for lunar return velocities) to one based on a series of benchmarks, all of which fall along the trajectory. It should be noted that, for all $\mathrm{V}<28.4 \mathrm{kft} / \mathrm{s}(8.65 \mathrm{~km} / \mathrm{s})$, the V8.65 radiative heating distribution is utilized. For the solution based on the full set of benchmarks, distributions are interpolated in velocity space from one benchmark to the next and the time-dependent reference heating and pressure values used to generate the dimensional results. For the purposes of this validation, the interpolated or "bridging" solution is assumed to represent the "best prediction" of the transient environments. The transient environments based on limited benchmark data are then measured against the bridging results in order to assess the degree of accuracy. Integrated heat loads as well as peak heat rate are assessed globally as well as at the location of maximum convective ("hot" corner) and radiative heating (stagnation area). The CAP database currently contains sufficient benchmark solutions to perform this validation for only one trajectory mode, the lunar ballistic return. Additional computational solutions for the LEO ballistic return profile were recently provided, enabling validation for the more benign LEO entry, where radiative heating is not significant. Figures $12 \mathrm{a}$ and $12 \mathrm{~b}$ illustrate the relevant benchmark solutions (filled circles) superimposed on the trajectories in altitude-velocity and AOA-velocity space, respectively. The point at $\sim 10 \mathrm{kft} / \mathrm{s}(3 \mathrm{~km} / \mathrm{s})$ was used in the bridging solution for both trajectories. Based on the altitude and velocity sensitivity studies described in the previous section, environments were also generated for each trajectory using the non-optimum (open circles) benchmark conditions identified in Fig. 12; V6, H44.5 and H65 for LEO, and V9, H48 and H65 for lunar return (see Tables 1a and 1b for equivalent English units conversion). Nonoptimum refers to the case where single benchmarks are selected that do not meet the "at or near" peak heating criterion.

Figure 13 illustrates the combined convective and radiative heating rate and load predictions for the hot corner location on the 28-deg lunar ballistic trajectory profile shown in Fig. 12. The heavy dashed line represents the interpolated result that bridges the solutions at each of the five benchmark conditions available along the profile. For this bridging solution, the case was set up to linearly interpolate the distribution between benchmarks. Distributions can also be held over a velocity range where appropriate. Again, the bridging (interpolated) prediction is assumed to represent the best possible solution using the present method to compute the transient environments. The lighter solid lines represent the use of each of the designated distributions throughout the entire trajectory rather than doing the bridging. Case designations in Fig. 13 use metric velocity per CAP ATDB. For corresponding H and AOA refer to Fig. 12. LEO case conditions are shown in Table 2. With the exception of the low-velocity benchmark conditions (LEO-323, 10 kft/s and V4, $13 \mathrm{kft} / \mathrm{s}$ ) that would not be expected to predict the peak heating levels, use of any of the individual benchmark cases yields results to within about $5 \%$ of the "best prediction" based on using five benchmark cases along the profile. Use of only the peak heating condition (V10.15), as recommended, captures the peak very accurately, yet results in only about $5 \%$ over-prediction of the load compared to the bridging result. Similar results are noted for the stagnation area. Figures $14 \mathrm{a}$ and $14 \mathrm{~b}$, also for the 28-deg lunar ballistic trajectory profile, show the relative contribution of the convective and the radiative heating components at the hot corner and the stagnation area, respectively. The radiative heating plays a more important role in the stagnation region than at the corner. In Figs. 14a and 14b, the solid lines represent the heating rate histories and the dashed lines the integrated heat loads. Only the bridging solution is shown in these figures. For the same trajectory profile, selection of a single

Table 2. LEO Case Designation

\begin{tabular}{cccccc}
\hline \hline Case & \multicolumn{2}{c}{ English } & \multicolumn{2}{c}{ Metric } & \multirow{2}{*}{ AOA } \\
Designation & Velocity, kft/s & Altitude, $\mathrm{kft}$ & Velocity, km/s & Altitude, $\mathrm{km}$ & \\
\hline LEO-185 & 24.795 & 246.522 & 7.56 & 75.14 & 27 \\
LEO-227 & 23.809 & 213.255 & 7.26 & 65.00 & 27 \\
LEO-260 & 21.527 & 185.581 & 6.56 & 56.57 & 27 \\
LEO-290 & 17.294 & 157.425 & 5.27 & 47.98 & 28 \\
LEO-323 & 9.907 & 121.873 & 3.02 & 37.15 & 30 \\
\hline \hline
\end{tabular}


benchmark solution at non-optimum conditions is examined in Figs. 15a (hot corner) and 15b (stagnation point) for the two altitude conditions previously identified in Fig. 12a. Here, the results based on the non-optimum benchmark conditions are compared to the "best prediction" based on the bridging solution. Using the $213 \mathrm{kft}(65 \mathrm{~km})$ benchmark case, which is extremely far away in trajectory space from the peak heating condition, the comparison to the bridging solution is within $4-8 \%$, with agreement to within $~ 5 \%$ in the stagnation area. A condition near peak heating is recommended to ensure the peak heating levels are captured. For the condition closer to peak heating, $157.5 \mathrm{kft}(48 \mathrm{~km})$, agreement is shown to within $5 \%$ of the bridging solution.

The ballistic LEO entry case was examined in much the same way. Benchmark conditions used for this case are shown in Table 2. Results are summarized in Fig. 16a for the hot corner, with excellent agreement shown between the single benchmark cases and the bridged solution with the exception of the low-velocity benchmark case $(\sim 10$ $\mathrm{kft} / \mathrm{s})$ as would be expected. Figures $16 \mathrm{~b}$ and $16 \mathrm{c}$ illustrate the resulting comparison when the non-optimum benchmark cases illustrated in Fig 12a are selected, for the hot corner and the stagnation point respectively. Agreement is shown to within 5\% on the corner, with 2-3\% agreement in the stagnation area, despite the use of the non-optimum benchmark cases.

Validation of the radiative heating predictions for the current methodology has been limited due to the limited set of benchmark solutions. The approach developed here will likely be used to assess the sensitivity of the TPS design to the large uncertainties in radiative heating predictions. The nature of the radiative heat pulse and the mode of entry, ballistic or skip, suggest that for most mission scenarios a substantial uncertainty in radiative heating can be tolerated for lunar return missions. However, return from Mars will likely be dominated by radiative heating, and it is critical that further research be done in this area.

\section{Implementation}

The methodology described here has been implemented in an advanced engineering environment and developed into an application known as K's Implicitly Simple Solution (KISS). The KISS application is built using TechnoSoft's Adaptive Modeling Language or AML. ${ }^{2{ }^{* *}}$ For this application, AML is run in a windows environment on a standard laptop computer using a single processor. AML is an object-based programming language whose "objects" are accessed by the engineer through a Graphical User Interface (GUI). For the KISS application, many of the objects are actually FORTRAN software applications developed over the years and adapted to operate within the AML environment. The KISS system is designed to operate with the engineer in the loop, and although it automates many of the tasks required to do the analyses described here, it cannot successfully generate reliable environments without a knowledgeable aerothermodynamicist in the loop. The KISS application has been developed to enable the rapid, yet accurate generation of aeroheating environments, not only to support a broad range of trade studies, but also to generate the data required for TPS design and analysis. The system can be used to rapidly transform a limited number of benchmark solutions into the transient environments required for thermal analysis; including heating rates and loads, surface pressures, radiation equilibrium temperatures, and shear stresses among others. While the intent of KISS is to generate those environments, the powerful geometry and visualization capability of the language also enables the rapid simulation of the environments in a visual display. The engineer interacts with the interface in one window to generate the models. Every analysis becomes a simulation. One window is set up to display the point mass of the vehicle as it follows the trajectory profile in altitude-velocity space and another simultaneously displays a visual simulation of the heating, temperature, pressure or shear stress environments. Figure 17a illustrates a screen capture of the application environment. This particular simulation shows a bridging model including five benchmark cases as indicated in the tree structure in the left-hand window and on the trajectory profile shown in the upper right. Environments are integrated over time, and global distributions of the maximum values over the entry profile are captured and can be easily displayed and evaluated in Tecplot ${ }^{2}{ }^{21}$ A time grab feature allows the engineer to assess the heating environments at any point along the trajectory, i.e. snapshots in time of the global distributions for all the heat-shield aeroheating environments. The radiative and convective components can be displayed separately or together. Color scales can be set in a meaningful way for the simulation. For instance, temperature bands can be selected to match TPS material capabilities. A restore feature allows the engineer to return and visualize the data so it is not necessary to rerun all the analyses in order to perform a simulation previously modeled. Several engineering features have been incorporated. A mapping technique ${ }^{22}$ is embedded in the tool so that computational solutions delivered on different meshes and in different coordinate systems can be mapped to a master grid and integrated into the analyses. The mapping process has been shown to retain the peak heating value to within $\sim 2 \%$ of the original data, for the mapping

\footnotetext{
${ }^{* *}$ Trade names and trademarks are used in this report for identification only. Their usage does not constitute an official endorsement, either expressed or implied, by the National Aeronautics and Space Administration.
} 
of the ATDB solutions from $\sim 9000$ point mesh to the $\sim 1000 \mathrm{pt}$ mesh used in this study. Numerous options have been included in the developmental version of the application to enable better error checking. The underlying benchmark data as well as the computed environments can be interrogated at any point in the analysis for any point on the heat-shield.

In addition to the heating rates that are generated, the engineer can choose how to define the heat-transfer coefficients, including the wall temperature and enthalpy assumptions. This capability was incorporated to accommodate the thermal analysis community and to better interface with not only the industry standard thermal tools such as SINDA, ${ }^{23}$ but also the ablation analysis codes like CMA. An option is also available to generate heating directly in the format required for EXITS, ${ }^{24}$ the thermal analyzer available as part of the MINIVER suite of tools, and also for TPS-it, ${ }^{25}$ a Langley-developed TPS analysis tool. The default reference parameter for convective heating rates is a Fay-Riddell based value derived from MINIVER, but a function of the form $\mathrm{k}_{1} \rho_{\infty}{ }^{{ }^{2}} \mathrm{~V}_{\infty}{ }^{\mathrm{k}}{ }_{3}$ is available where the user can prescribe the coefficients or use the ones built in to generate a Sutton and Graves ${ }^{13}$ number for use as an alternate reference value.

KISS is designed to accommodate metric/English input/output in any combination. The underlying tools run in their native units and transformations are handled at the input/output stage of the process. In order to set up a simple model within the KISS application, the engineer performs 5 steps ( 6 for a bridging/interpolation model): 1) select a master mesh (to which all input computational solutions will be mapped, if not already on a common mesh), 2) select a convective solution input file, (also a radiative solution input if applicable, or a simple stagnation point equilibrium radiation calculation can be used), 3) select a trajectory file 4) choose an atmosphere model - 1976 standard atmosphere ${ }^{26}$ is the default, and 5) input the freestream/velocity/emissivity condition at which the benchmark case was computed. If an interpolation model is selected the user must select all the input solutions files and set the reference conditions for each. In this case, the $6^{\text {th }}$ step is to set the velocity break points at which the engineer wishes to transform from one distribution to the next. Figures 17-19 illustrate the bridging capability and verify the implementation in the KISS application. Figures $17 \mathrm{~b}$ and $17 \mathrm{c}$ show the well-behaved transformation (or "morphing") in convective and radiative distribution along the symmetry plane over the user-selected range for the model illustrated in Fig. 17a, the 28-deg lunar ballistic return. Figure 18 shows the associated reference heating profiles which, when used with the non-dimensional distributions shown in $17 \mathrm{~b}$ and $17 \mathrm{c}$, produce the dimensional heating rates shown in Figs. 19a-19c, for convective, radiative and combined values respectively. It is clear from examination of these profiles that the desired bridging approach has successfully been implemented within the design procedure in the AML environment.

Run times for the KISS application range from a couple of minutes for a single simulation on a coarse mesh of about 1000 nodes and a trajectory on the order of 900 seconds (at 1 second intervals) to a 30-45 minutes for a simulation with five benchmark conditions, and a 2000 second trajectory on a comparable mesh. Times are quoted based on a 1.66 Ghz, Centrino Core Duo Processor. At present, the KISS application is not run in parallelized environment, and each of the interpolation cases is run sequentially, thus accounting for the long run time. A typical analysis for the same $1000 \mathrm{pt}$ mesh and 2000 second profile with five benchmark analyses, generates about 1.5 GB of data, the majority of which is saved in order to enable the on-demand simulation in the restore mode. Analysis files are output in Tecplot ${ }^{\circledR}$ format and can be generated for a user-defined node set which can reduce the file size substantially. Nodes can be automatically selected along the symmetry plane, all nodes within a given radius of an input $\mathrm{x}, \mathrm{y}, \mathrm{z}$ location, or selected interactively. Simulations are run in a demand driven mode, i.e. if the user requests a convective simulation, the code will do only the calculations required to generate that simulation. If a radiation equilibrium temperature simulation is requested, the convective and radiative heating will first be generated in order to display the radiation equilibrium temperature. An option to "generate all files" is available. Once all these data are generated any individual simulation can be run quickly depending upon the number of time steps required and the simulation delay time set by the user. The simulation can be run in real time, but for cases such as the lunar skip return trajectories with durations on the order of 30 minutes or more, this is impractical.

\section{Trade Studies}

Several trade studies have been performed using this approach. A few are highlighted here to illustrate the power of this method for the rapid generation of aeroheating environments.

\section{A. Lunar Ballistic Return (LBR) versus Lunar Skip Return (LSR) - "28-deg" trim AOA}

One of the issues NASA must determine is the nominal entry mode for return from the moon. The two major options are a direct entry, lunar ballistic return and a "skip" entry where the vehicle dives into the atmosphere and skips back out and then returns on what is essentially a LEO return profile. See Fig. 4 for a comparison of the 
profiles. The main advantage of the skip is that it allows the controllers to extend the range and offers many more desirable landing opportunities and locations. On the other hand, the direct ballistic return allows little latitude in landing locations once the commitment is made to return in this fashion. Both modes offer unique, but different challenges for the TPS design. On the skip entry, the TPS will see a double heating pulse separated by a substantial cool-down as the vehicle returns to space. Thus, an already ablated TPS will be returning through a secondary heating pulse. The first pulse on the skip entry will be on the order of that for the ballistic entry, but the longer duration and the second pulse will result in substantially higher heat loads for the skip entry compared to the ballistic.

Figure 20a compares the peak heating rates on the ballistic and skip entries for a nominal trim AOA of 28 deg. As noted previously the 28-deg designation is an ESAS legacy value, but the trim AOA is really on the order of 26.5 deg. Figure $20 \mathrm{~b}$ compares the global distribution of the total heat load over each entry. The skip trajectory is illustrated in the top half of the figure and the ballistic on the lower half. While it is clear from these figures that the rates are not noticeably different, there is a dramatic increase in load for the skip return. For an ablative system much of the energy is transformed through the ablation mechanism so load does not directly translate into weight, but nonetheless it would be reasonable to assume that the heat-shield for the skip would necessarily weigh more that one designed strictly for a ballistic return.

\section{B. LEO Return - Fully Catalytic versus Non-Catalytic wall Boundary Condition}

When the Shuttle orbiters are retired in 2010, the United States could be left without the capability to deliver and return the International Space Station (ISS) crew. Although government money has been set aside to encourage commercial developers to fill this gap, the CEV is also being considered for the ISS missions. While the environments for return from a LEO/ISS mission are significantly less severe than for return from the moon, the cost of developing two unique TPS concepts, if not heat-shield support structures, may be prohibitive. The ablative heatshield development program may not be sufficiently mature in time, and there are also ongoing discussions concerning the ablator performance characteristics in this more benign environment. If the ablative material is utilized in an environment in which it does not ablate, much of the advantage may be lost. Coupled with the ISS return as the more immediate mission need, designers are also considering the application of Shuttle-like tiles for the ISS mission. To date, all the solutions that constitute the CEV aerothermal database are predicated on a fully catalytic wall boundary condition. The CEV fully catalytic laminar heating rates and the associated temperatures that are computed for the LEO entry exceed the capability for the Shuttle tiles. However, the argument can been made that since these tiles are coated with a reaction cured glass (RCG) which is far less catalytic, the heating rates may actually fall to lower levels much more amenable to the tile concepts.

Although the present methodology has not been validated against any non-catalytic heating data, it is anticipated that the assumptions inherent to the method will also hold for a non-catalytic boundary condition (although likely not for reaction rates in between as for the RCG coating). Nonetheless, the non-catalytic values are expected to provide a lower bound for the rates, and thus the temperatures. With this in mind, a trade was performed using the KISS procedure and the benchmark conditions for which convective solutions are available for a ballistic return from LEO (four for non-catalytic and five for fully catalytic). The non-catalytic benchmark cases for the conditions shown in Fig. 12, are not included in the aerothermal database, but were recently generated by the CAP team to explore the wall boundary condition and its impact on tile surface temperatures. Figure 21a illustrates the potential reduction in radiation equilibrium temperature (note Rankine values) associated with the change in wall boundary condition from catalytic to non-catalytic, with the non-catalytic results shown in the upper half of the figure. Radiation equilibrium temperature is a good representation of wall temperature for a high performance insulator such as a Shuttle tile.

The single-use temperature quoted for the high-temperature Shuttle tile is $\sim 3200 \mathrm{R}$. Slumping of the tile can be expected to begin at somewhat lower temperatures. Although, as evident in Fig. 21a, the non-catalytic surface is shown to reduce the temperature levels compared to the fully catalytic values, much of the heat-shield surface would see temperatures exceeding $3000 \mathrm{R}$, with temperatures greater than $3200 \mathrm{R}$ in the corner region. Figure $21 \mathrm{~b}$ illustrates the corresponding shear stress-distribution with the highest values at the hot corner as expected. The potential slumping due to high temperature, particularly in the presence of high shear suggests that the reduction in heating, even for a non-catalytic surface, is insufficient to allow the application of a typical Shuttle tile for the CEV return from LEO.

\section{Trim AOA Assessment - " $28 \mathrm{deg}$ " versus 20-deg AOA}

Initial planning for the lunar return mission suggested many advantages to be gained if a trim $\mathrm{L} / \mathrm{D}$ on the order of 0.4 could be achieved. For reference, the Apollo L/D in the same speed regime was on the order of a little over 0.3. 
The additional crossrange from a higher $\mathrm{L} / \mathrm{D}$ increases $\mathrm{CEV}$ landing options for all missions and, according to ESAS, would enable CONUS landings when returning from ISS. Thus the aerothermal database and the baseline entries were developed based upon a trim AOA in the neighborhood of $28 \mathrm{deg}$. However, the vehicle could not be packaged to obtain the desired trim L/D. Recent developments suggest that, while the trim AOA may not be as low as $20 \mathrm{deg}$, it will nonetheless decrease from the assumed value of $28 \mathrm{deg}$. The reduced trim AOA has far-reaching implications. The landing opportunities and options will be more limited. Both the aero and the aerothermal database were built around a higher AOA, so additional benchmarks may be needed to fill in the gaps. Heating distributions over the vehicle will change with the reduced AOA. For the lunar return missions, the relative contribution and levels of convective and radiative heating might change.

Trajectories with a $\sim 20$-deg trim AOA have recently been provided. These entries offer an opportunity to begin to investigate the heating and TPS implications associated with the lower AOA schedule. A comparison of the "28deg" and the 20-deg AOA schedules is illustrated in Fig. 3. As observed previously, the AOA in both cases remains fairly constant over the period of peak heating. The corresponding altitude-velocity profile comparison is shown in Fig. 2. For the case of the lunar ballistic entry, it is observed that the flight profiles are nearly identical once the vehicle reaches pullout at around $180 \mathrm{kft}$, but the 20 -deg profile enters from a higher energy state. For the purposes of this assessment, the 20-deg lunar ballistic entry profile was used with the vehicle attitude held at 28 and 20 deg respectively to assess the heating implications associated with the lower trim AOA. The 1976 standard atmosphere model is also assumed in both cases.

Figures $22 \mathrm{a}$ and $22 \mathrm{~b}$ illustrate the heat rate and the integrated heat load comparison for the recently acquired lunar ballistic trajectory for the vehicle flying an attitude of $28 \mathrm{deg}$ vs $20 \mathrm{deg}$. In other words the vehicle is assumed to follow the same profile in altitude velocity space, yet oriented at a different attitude. The 28-deg entry is shown in the upper half in each figure and the 20-deg entry in the lower half. The influence of the radiation for the lower trim AOA is clear, as evidenced by the heating pattern showing higher values over the subsonic region (stagnation area). The contribution due to convection is noted as the "crescent moon shape" on the hot side. The components of the heating are shown in Figs. 22c and 22d for convection and radiation respectively. Note the different scales in these two figures. As expected, the convective heating levels shown by the contours are observed to decrease with decreased AOA, and the radiative levels are observed to increase with decreased AOA. Radiative heating patterns generally appear similar to a pressure distribution with the hot spot centered at the stagnation point. In each case, the data are consistent with an increase in effective radius associated with the reduced AOA. For the lower trim AOA, the influence of radiation has apparently become more dominant, but large uncertainties are associated with the radiative heating calculations. In fact, the program margin plan calls for a factor of two to be placed on the radiative predictions to account for this uncertainty. For the higher trim AOA entries, program studies had shown that a factor of two on the radiation did not have much, if any, impact on the TPS design primarily due to the short duration of the pulse. Figures 23a and 23b compare the rates and loads respectively for the same 28 and 20-degree trim AOA profiles used in Figs. 22a and 22b, but with a 100 percent margin applied to the radiative heating component (doubling the value). The pattern of the heating suggests that the rate and load are dominated by radiation for the 20deg profile whereas for the 28 -deg profile more of the crescent-shaped convective pattern remains evident.

\section{20-deg Lunar Ballistic Return - 1976 Standard Atmosphere versus GRAM-99 ${ }^{27}$}

As the program matures, the GN\&C (Guidance, Navigation and Control) team has begun to transition to a more accurate atmospheric model, using a GRAM-99 model rather than the 1976 standard. The GRAM-99 includes seasonal dependence, geographical dependence, ability to model uncertainties, etc. In the current method, the freestream conditions applicable to the benchmark solutions are used to non-dimensionalize the distributions. The dimensional heating rates are recovered over the trajectory profile by using the atmosphere model associated with any given trajectory to generate the transient reference values. Therefore, this approach can be used to examine the implication of different atmosphere models on the heating levels. It is not known if the current program methodology based on Mach, dynamic pressure, and AOA space is uniquely defined to account for atmosphere model changes. The 20-deg trajectory profiles were generated with a GRAM-99 atmosphere model for the date $1 / 10 / 10$. Figures $24 \mathrm{a}$ and $24 \mathrm{~b}$ compare the heating rates and integrated heat loads, respectively, for this trajectory for which the environments were run first with the as-built GRAM atmosphere and then with the 1976 standard atmosphere. The upper half of each figure shows the results based on the GRAM-99 atmosphere model, and the lower those based on the 1976 standard. The atmosphere model selection is seen to have a significant influence on both the heat rate and load. Currently, there are several strategies for TPS sizing and design, and it is not known how or if these heating differences due to atmospheric models may affect those plans. This trade was performed to highlight the potential influence of the atmosphere model on heating rates and loads. The particular results, are highly dependent upon the time of year selected. 


\section{E. Lunar Skip Return - 20-deg - variable atmosphere implications}

A trade study will not be performed to examine this topic, but it should be noted that there are unique issues associated with the GRAM-99 atmosphere, or any other non-standard model for the skip-type entries. The aeroheating community typically works with an atmosphere that is uniquely defined through the altitude range, but does not have a time-dependency. For the skip trajectories, the vehicle essentially enters for the first time in one part of the world, skips up to about $450 \mathrm{kft}$, and descends again from there. For its second "entry" the vehicle is half way around the world from the initial entry and now traversing through an entirely different atmosphere. These are not the standard atmospheric dispersions with which the aeroheating community is typically concerned. The temperature of the atmosphere can have a significant effect on the vehicle heating levels as demonstrated by the comparisons in the previous section. Figure 25 shows the freesteam temperature and pressure as an integrated function of altitude for the complete trajectory and for just the second pass. The solid lines represent the second pass and pressure and temperature are uniquely defined for a given altitude on that pass. When the atmosphere of the first pass and the skip out are combined with the second pass, the differences in the atmosphere are observed as what appears to be high frequency oscillations in the profile. In order to adequately model the atmosphere for the skip cases (the one using a GRAM atmosphere), a time-dependent capability would need to be added. In the interim, the trajectories can be run with the atmosphere for each phase of the skip (down, up, down). The integrated loads and the peak heat rates can be determined for each phase using the proper portion of the atmosphere. An evaluation against the results using the "combined" atmosphere will establish the sensitivity of the results, and thus determine the requirement for adding a time-dependent atmosphere for the transient aeroheating analyses.

\section{Limitations}

As noted, the method described here does not explicitly compute any surface heating rates without a minimum of one benchmark solution at or near peak heating for the trajectory of interest. Currently, only data for fully catalytic, laminar heating rates have been validated in the methodology. The predictions based on this procedure are entirely reliant on the quality of the incoming benchmark solutions, both convective and radiative. At this time, the simplified method has only been demonstrated for application to an Apollo-like configuration, and results are limited to the forebody heat shield. The present implementation is based on trajectories with a fairly constant trim AOA. Sufficient benchmark cases must be available to adjust for the effect of AOA on heating along the profile. Currently the CEV is being designed for all-turbulent heating, and the turbulence issue has yet to be addressed in the current methodology. An engineering approach based on wind tunnel data doubled the corresponding laminar rates for use in the Smart Buyer study.

\section{Future Enhancements/Alternative Applications}

Several enhancements are currently under consideration to extend the capability to other applications, including perhaps planetary entry at Mars. Planetary vehicles tend to maintain a constant trim AOA, so the enhancements required would be mainly the addition of a $\mathrm{CO}_{2}$ atmosphere capability and adaptation of the appropriate reference heating models. For the current application, an engineering approach to adapt the method to larger variations in AOA is being considered. Similarly, an engineering approach to allow the generation of turbulent heating environments will likely be explored. The method has been developed with sufficient flexibility to allow generation of the environments in the format required for various existing TPS analysis tools. It is hoped that those TPS tools will soon be adapted to the AML environment and the capability will be extended to provide a heat-shield thermal analysis and sizing, at least for a non-structural heat-shield element. Environments/loads generated can also support heat-shield, carrier structure design, and thermostructural analyses.

\section{Concluding Remarks}

The simplified method for producing transient heating environments for Apollo-like configurations was shown to provide reliable results for both heating rates and loads, and its implementation in the KISS (K's Implicitly Simple Solution) application offers a valuable tool for the performance of a wide range of Thermal Protection System/vehicle trade studies. Inherent to the approach is the ability to allow the rapid assessment of heat-shield environments as the vehicle, the missions and thus the entry trajectories evolve. The method was initiated in support of the CEV (Crew Exploration Vehicle) Smart Buyer Activity and is intended to complement the CEV project's current procedure for producing the vehicle surface heating rates and loads throughout the entry trajectory. Corresponding values of the local shear stresses and pressures can also be obtained. The present approach does not predict the heating without benchmark convective and radiative solutions. Computational fluid dynamic-based fully catalytic, laminar heating rates together with solutions from a benchmark radiation code are non-dimensionalized

12

American Institute of Aeronautics and Astronautics 
with the appropriate reference values. For a trajectory with five benchmarks cases, distributions are interpolated in velocity space from one benchmark to the next with the time-dependent reference heating, pressure and shear stress values used to generate the dimensional results. For the purposes of the paper, the interpolated or "bridging" solution was assumed to represent the "best prediction" of the transient environments for the present technique. The transient environments based on fewer benchmark cases were then measured against the interpolated/bridged results in order to assess the degree of accuracy. It was demonstrated that a benchmark case could be selected at or near peak heating and used to determine the transient environments for the trajectory with discrepancies of less than about $10 \%$ in heating rate or load compared to the "best" solution. For this new approach, there is no requirement for a large CEV aerothermal benchmark database. The method provides a unique capability for conducting a wide range of trade studies. The utility of the technique for trade/sensitivity studies was demonstrated by performance of several quick-look assessments, including the impact of a trim angle-of-attack change from $\sim 28 \mathrm{deg}$ to $20 \mathrm{deg}$, and the relative impact of the uncertainty in radiative heating, the use of a GRAM atmosphere model compared to a 1976 standard, entry trajectory mode comparison for lunar return - skip vs ballistic - and LEO environments for fully catalytic vs noncatalytic wall boundary conditions. Of particular note is the speed and ease with which trades such as those illustrated here can be accomplished.

\section{Acknowledgments}

The authors would like to thank several people who contributed to the success of this effort, particularly in light of the heavy load they are already carrying in support of several other activities; Frank Greene of the Aerothermodynamics Branch who provided the LEO solutions that aided in the validation of the approach and enabled the catalycity trade study, Brian Hollis, also of the Aerothermodynamics Branch, who provided the RADEQUIL solutions that led us to understand the need to extend the range of the radiative reference parameter, and finally to Ken Sutton of the National Institute of Aerospace who provided the extension to his original air radiation lookup tables. In addition we would like to extend our appreciation to Randy Lillard of the CAP team who made access to the ATDB benchmark solutions possible for us. Finally, we would like to thank Anne Rhodes for her illustrator efforts without whom the paper would be unreadable and the figures nonexistent.

\section{References}

${ }^{1}$ Kinney, David J., “Aerothermal Anchoring of CBAERO Using High Fidelity CFD”, CEV Aerosciences Project document, $\sim$ Feb 2006.

2“Exploration Systems Architecture Study - Final Report," Exploration Systems Mission Directorate, NASA TM-2005214062, November 2005.

3“Crew Exploration Vehicle "Smart Buyer" Design Team Final Report," NASA Engineering And Safety Center, May 2006.

${ }^{4}$ Curry, D.M., and Stephens, E.W., "Apollo Ablator Thermal Performance at Superorbital Entry Velocities," NASA-TND5969, Sept. 1970.

${ }^{5}$ Gnoffo, P.A., "An Upwind-Biased, Point-Implicit Relaxation Algorithm for Viscous, Compressible, Perfect-Gas Flows". NASA TP-2953, Feb. 1990.

${ }^{6}$ Candler, G.V., Wright, M. J., and McDonald, J. D., "Data-Parallel Lower-Upper Relaxation Method for Reacting Flows," AIAA Journal, Vol. 32, No. 12., 1994, pp. 2380-2386.

7"Development of the Crew Exploration Vehicle Aerothermodynamic Design Database," CEV Aerosciences Project, Aerothermodynamics Team, CAP TN EG-CEV-06-9, Feb. 2006.

${ }^{8}$ Whiting, E.E., Park, C., Liu, Y., Arnold, J.O., and Paterson, J.A., NEQAIR96, Nonequilibrium and Equilibrium Radiative Transport and Spectra Program: User's Manual, NASA RP 1389, Dec. 1996

${ }^{9}$ Fay, J.A., and Riddell, F.R., "Theory of Stagnation-Point Heat Transfer in Dissociated Air," Journal of Aerospace Sciences, Vol. 25, No. 2, 1958, pp. 73-85,121.

${ }^{10}$ Engel, C. D. And Praharaj, S. C., "MINIVER Upgrade for the AVID System, Vol. I: LANMIN User's Manual," NASA CR-172212, Aug. 1983.

${ }^{11}$ Hoshizaki, H. and Wilson, K.H., "Convective and Radiative Heat Transfer During Superorbital Entry," AIAA paper 66106, Jan. 1966

${ }^{12}$ Cohen, N.B., " Boundary-layer Similar Solutions and Correlation Equations for Laminar Heat-Transfer Distribution in Equilibrium Air at Velocities up to 41,100 Feet Per Second," NASA-TR-R-118, 1961.

${ }^{13}$ Sutton, K., and Graves, R.A., Jr., "A General Stagnation-Point Convective-Heating Equation for Arbitrary Gas Mixtures," NASA TRR-376, 1971.

${ }^{14}$ Sutton, K., and Hartung, L.C., "Equilibrium Radiative Heating Tables for Earth Entry,” NASA TM-102652, May 1990.

${ }^{15}$ Tauber, M.E., and Sutton, K., "Stagnation-Point Radiative Heating Relations for Earth and Mars Entries," Journal of Spacecraft and Rockets, Vol. 28, No. 1, Jan.-Feb., 1991, pp. 40-42.

${ }^{16}$ Nicolet, W.E., "User's Manual for the General Radiation Transfer Code (RAD/EQUIL)". NASA-CR-116353, Oct. 1969. 
${ }^{17}$ Anon, "User's Manual, Aerotherm Charring Material Thermal Response and Ablation Program,” Acurex UM-87-13/ATD Acurex Corporation, Aerotherm Division, Mountain View, California, Nov. 1987.

${ }^{18}$ Chen, Y.K., and Milos, F.S., "Ablation and Thermal Response Program for Spacecraft Heatshield Analysis,", Journal of Spacecraft and Rockets, Vol. 36, No. 3, May-June, 1999, pp. 475-483.

${ }^{19}$ Ried, R.C., Jr., Rochelle, W.C., and Milhoan, J.D., "Radiative Heating to the Apollo Command Module: Engineering Prediction and Flight Measurement," NASA TM X-58091, April 1972.

${ }^{20}$ Anon, “Adaptive Modeling Language, Version 3.3.2, Reference Manual," TechnoSoft, Inc., 2002.

${ }^{21}$ Anon, Tecplot ${ }^{\circledR}$, User's Manual, Version 10, March 2005.

${ }^{22}$ Samareh, J. A., and Bhatia, K.G., "A Unified Approach to Modeling Multidisciplinary Interactions”, AIAA-2000-4704, Sept. 2000.

${ }^{23}$ Anon., "SINDA/FLUINT User's Manual, Version 4.1," Cullimore and Ring Technologies, Inc., Oct. 1998.

${ }^{24}$ Pond, J.E., and Schmitz, C.P., "MINIVER Upgrade for the AVID System, Vol. III: EXITS User's and Input Guide," NASA CR-172214, Aug. 1983.

${ }^{25}$ Plassman, G.E., "Phase Two TPS-it Code Final Report,” Raytheon Technical Services, NASA TO L-70750D, June 2003.

${ }^{26}$ U.S. Standard Atmosphere, National Oceanic and Atmospheric Administration, NASA and U.S. Air Force, Washington, DC, Oct. 1976.

${ }^{27}$ Justus, C.G. and Johnson, D.L., "The NASA/MSFC Global Reference Atmospheric Model - 1999 Version (GRAM-99)," NASA TM-1999-209630, May 1999. 


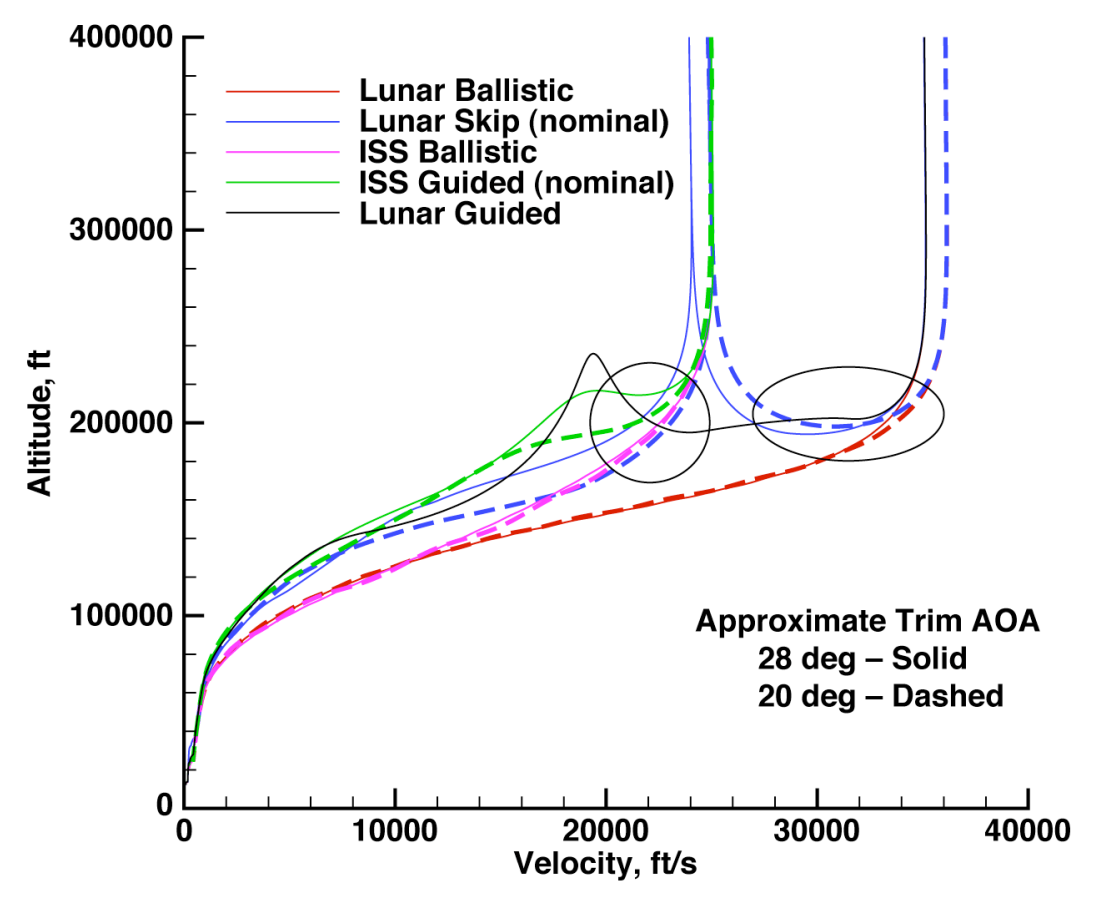

Figure 2. Altitude-Velocity Space - Variation with Trim AOA.

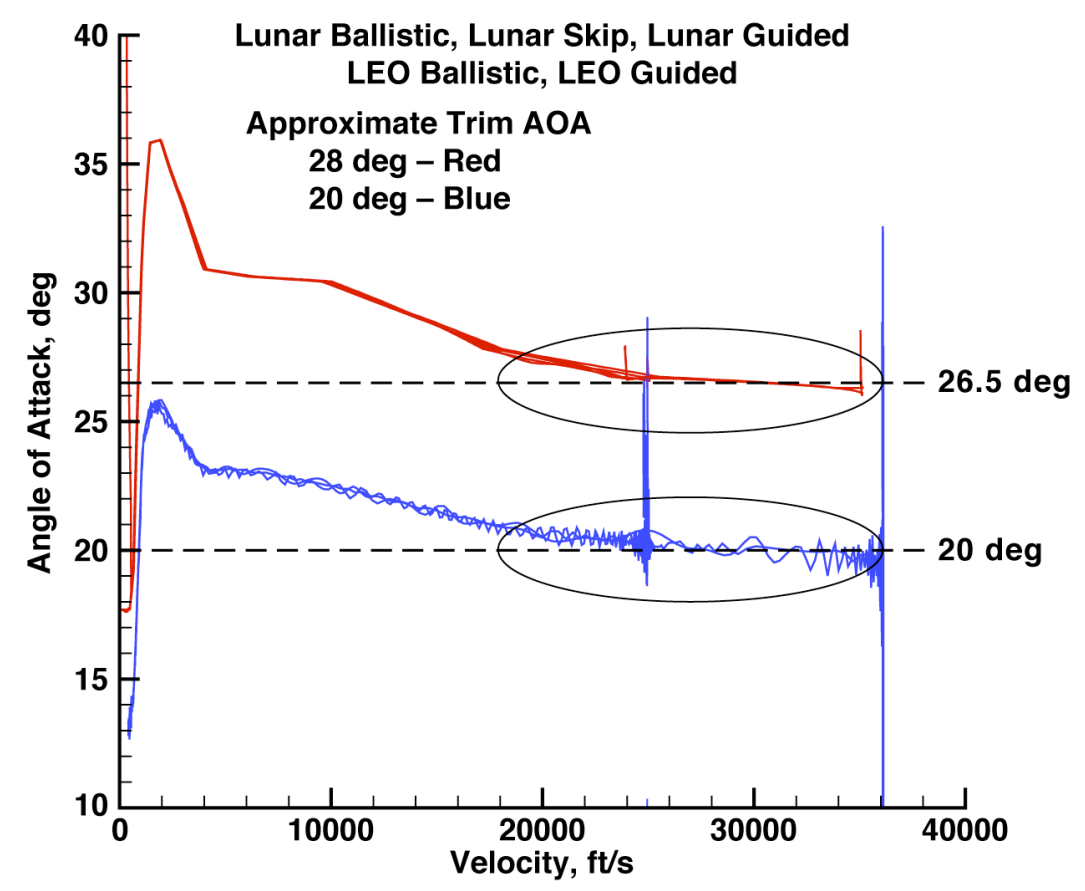

Figure 3. Angle of Attack - Velocity Dependence. 


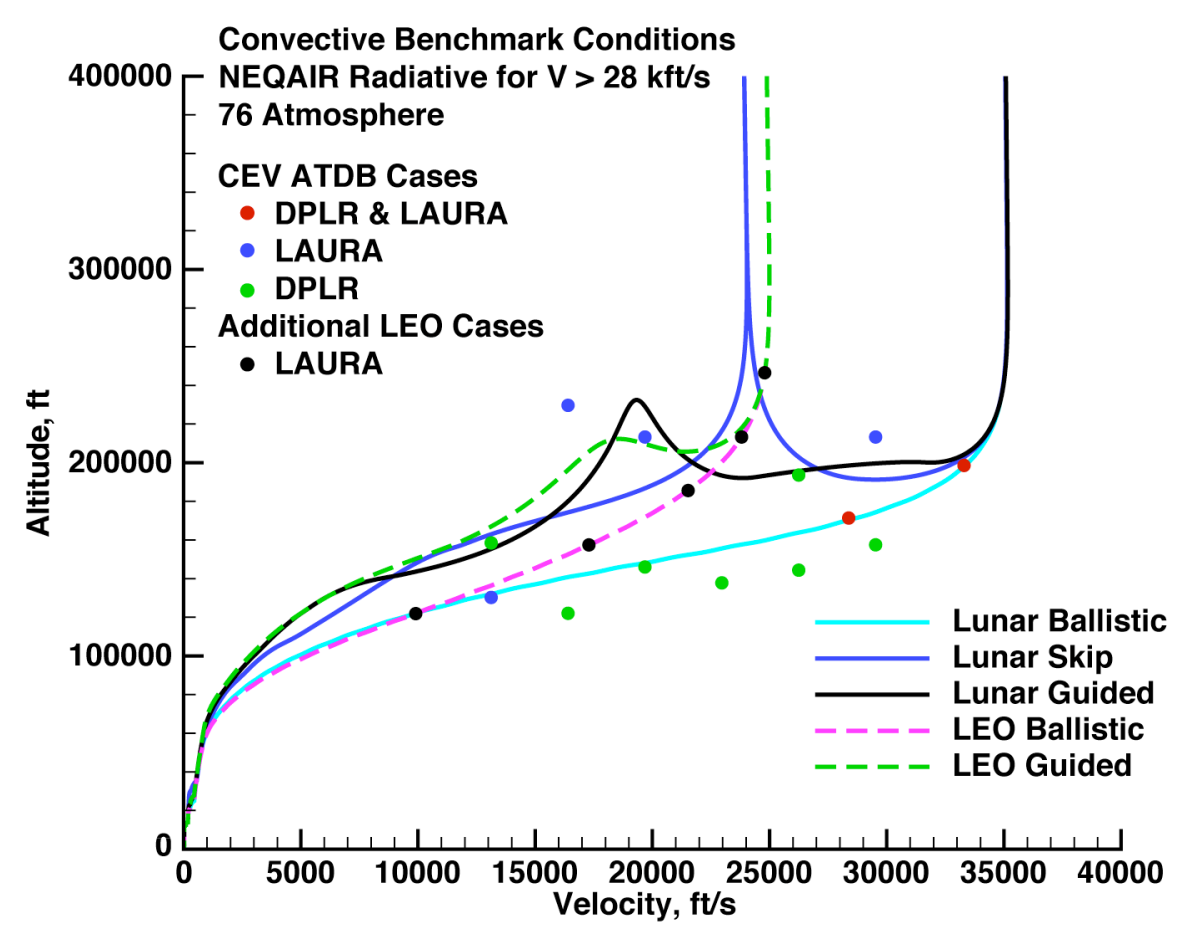

Figure 4. Altitude-Velocity Space - Benchmark Conditions.

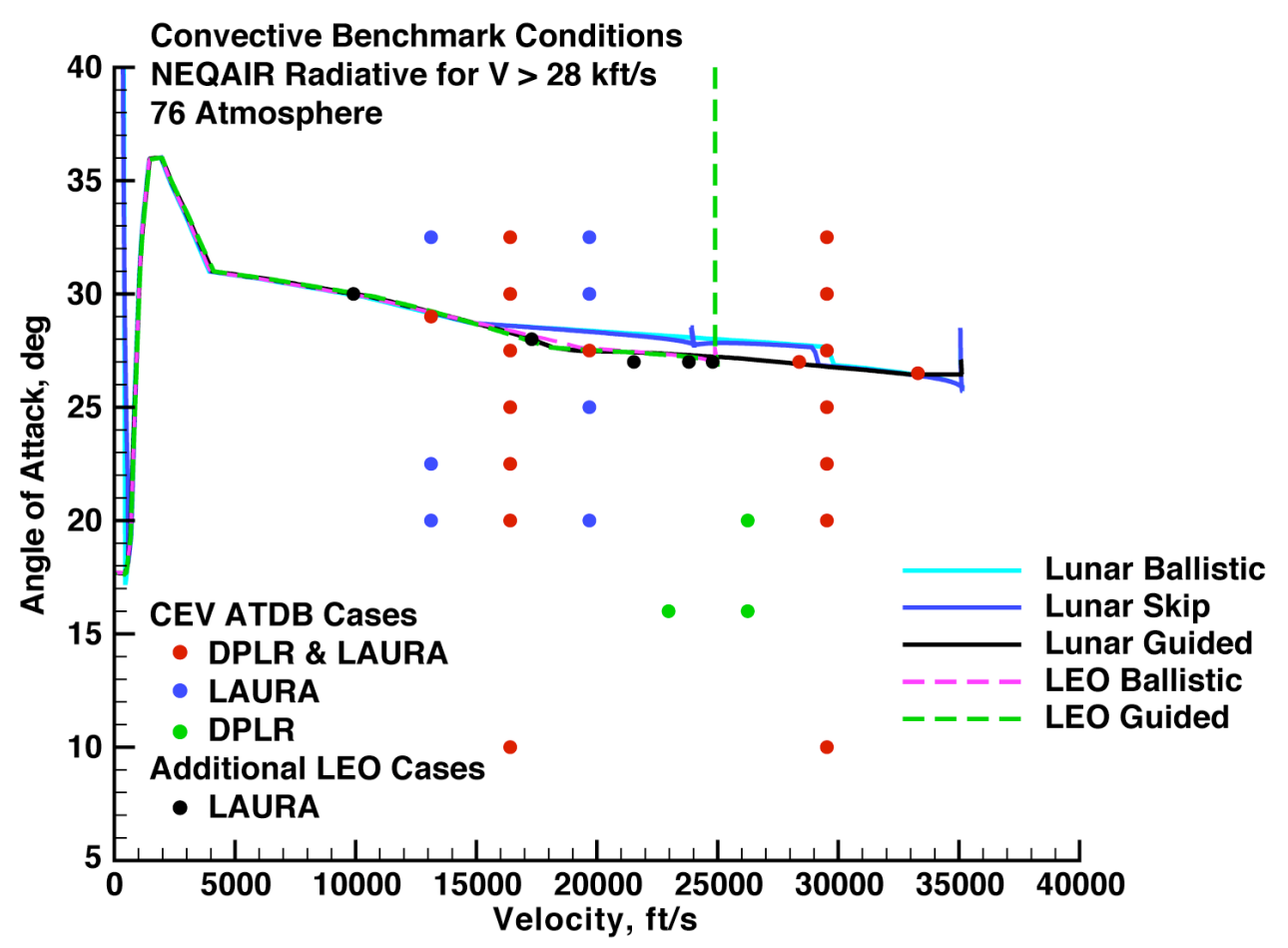

Figure 5. Angle of Attack-Velocity Space - Benchmark Conditions. 


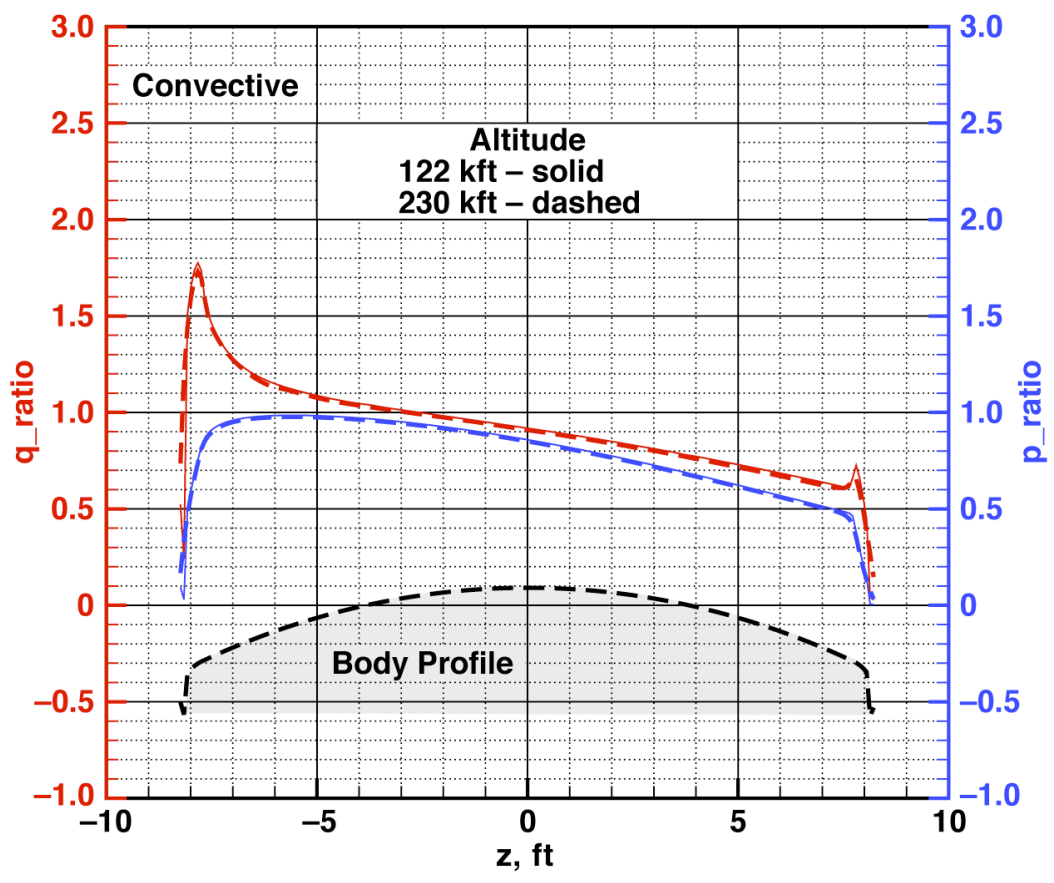

Figure 6(a). Symmetry Plane Heating/Pressure Distributions - Altitude Sensitivity; $\mathrm{V}=16.4 \mathrm{kft} / \mathrm{s}, \mathrm{AOA}=20 \mathrm{deg}$.

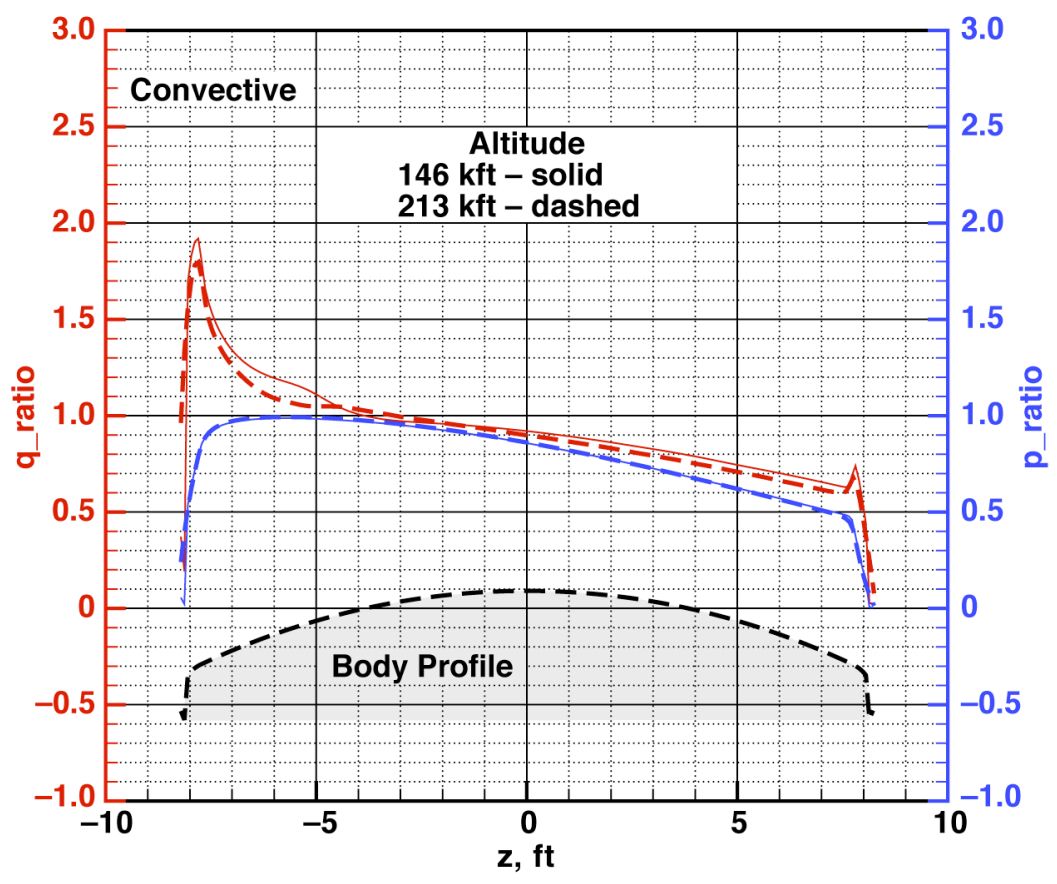

Figure 6(b). Symmetry Plane Heating/Presure Distributions - Altitude Sensitivity; $\mathrm{V}=19.7 \mathrm{kft} / \mathrm{s}, \mathrm{AOA}=20 \mathrm{deg}$. 


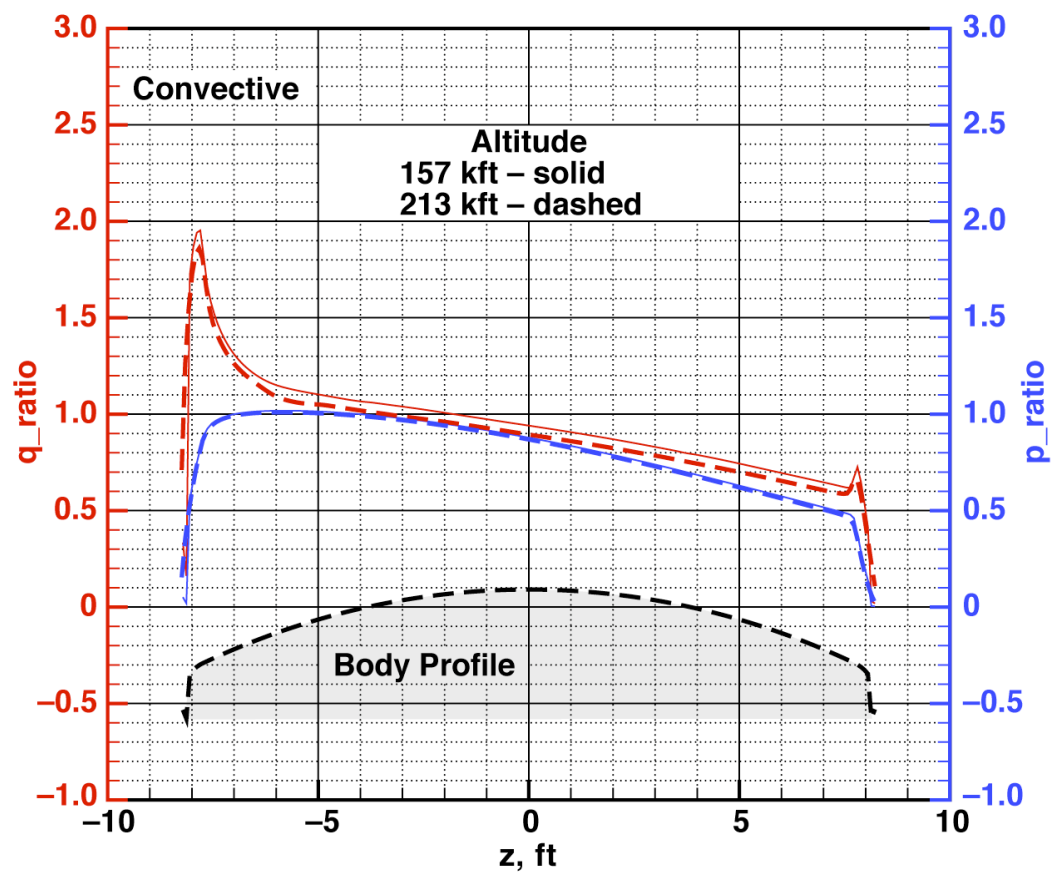

Figure 6(c). Symmetry Plane Heating/Pressure Distributions - Altitude Sensitivity; $\mathrm{V}=29.5 \mathrm{kft} / \mathrm{s}, \mathrm{AOA}=20 \mathrm{deg}$.

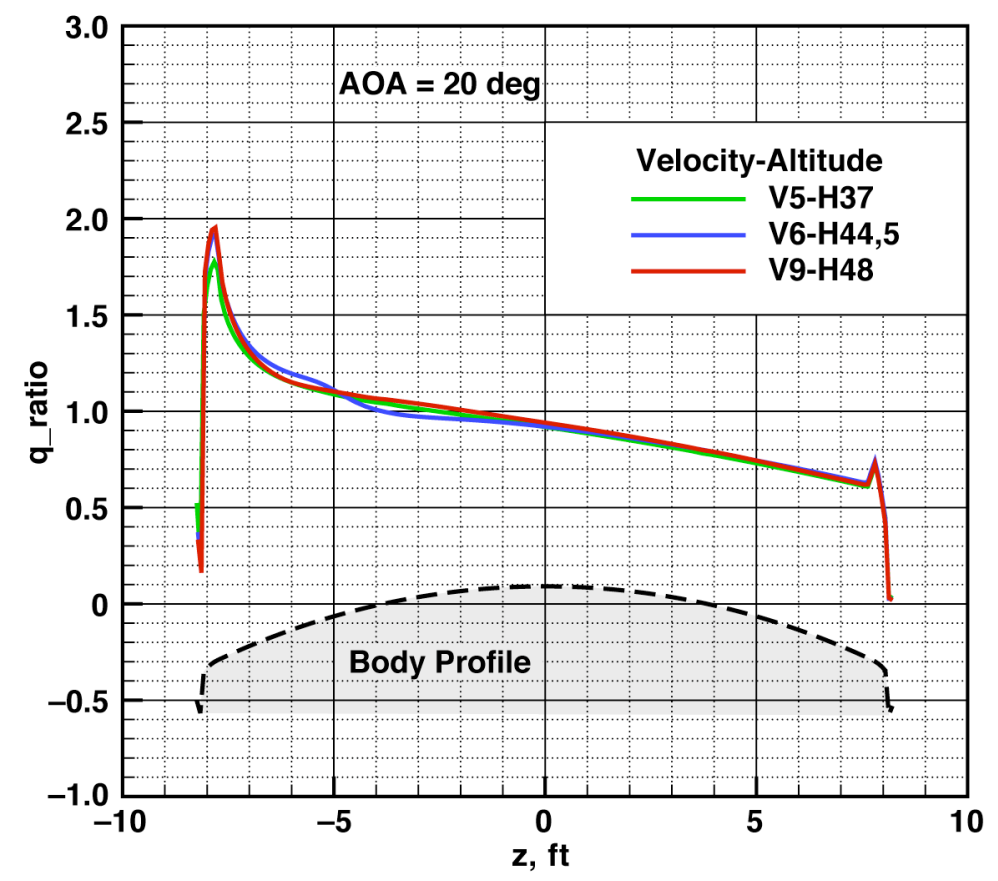

Figure 7. Symmetry Plane Convective Heating Distribution - Velocity Sensitivity; AOA-20 deg. 


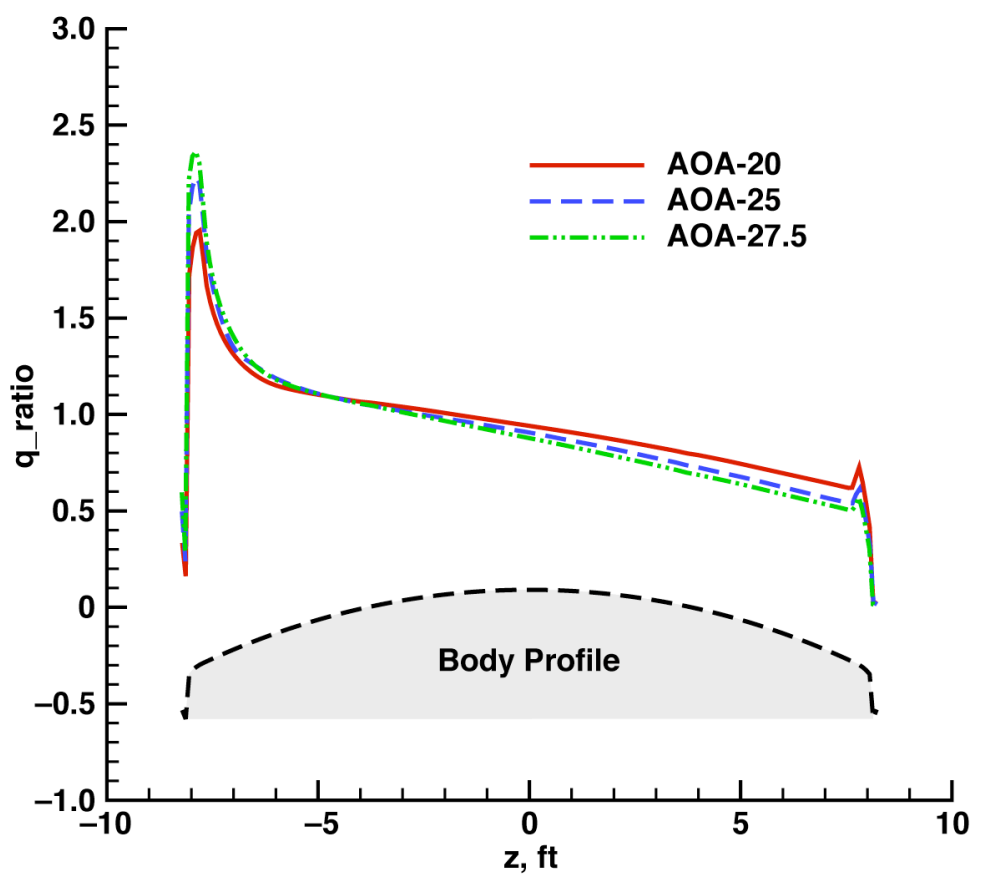

Figure 8(a) Convective Heating Distribution - AOA sensitivity; $\mathrm{V}=29.5 \mathrm{kft} / \mathrm{s}, \mathrm{H}=157 \mathrm{kft}$.

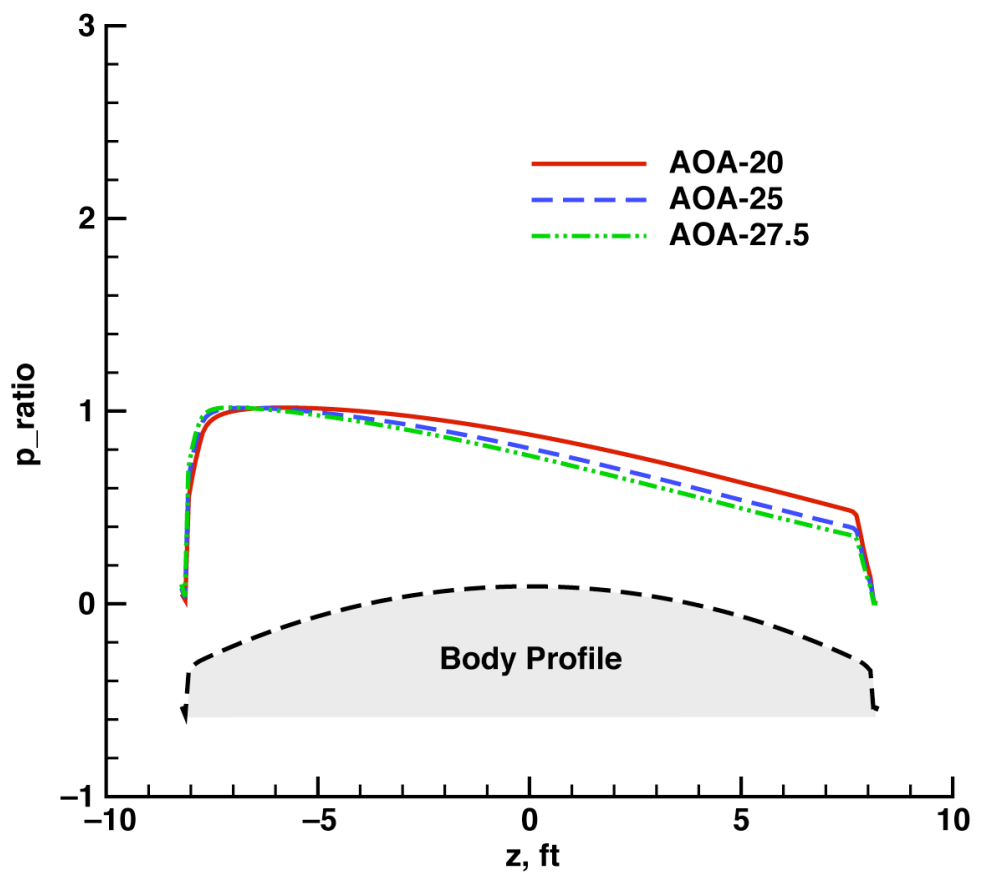

Figure 8(b). Pressure Distribution - AOA Sensitivity; $\mathrm{V}=29.5 \mathrm{kft} / \mathrm{s}, \mathrm{H}=157 \mathrm{kft}$. 


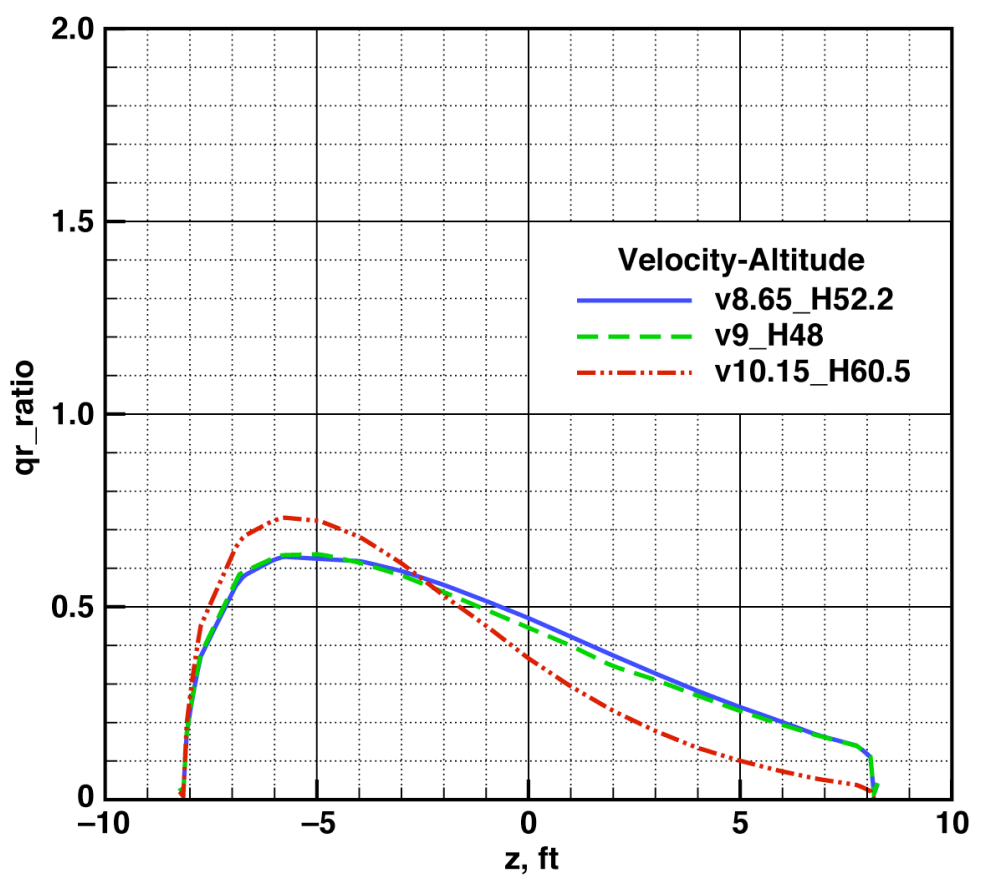

Figure 9. Radiative Heating Distribution - AOA 27 deg.

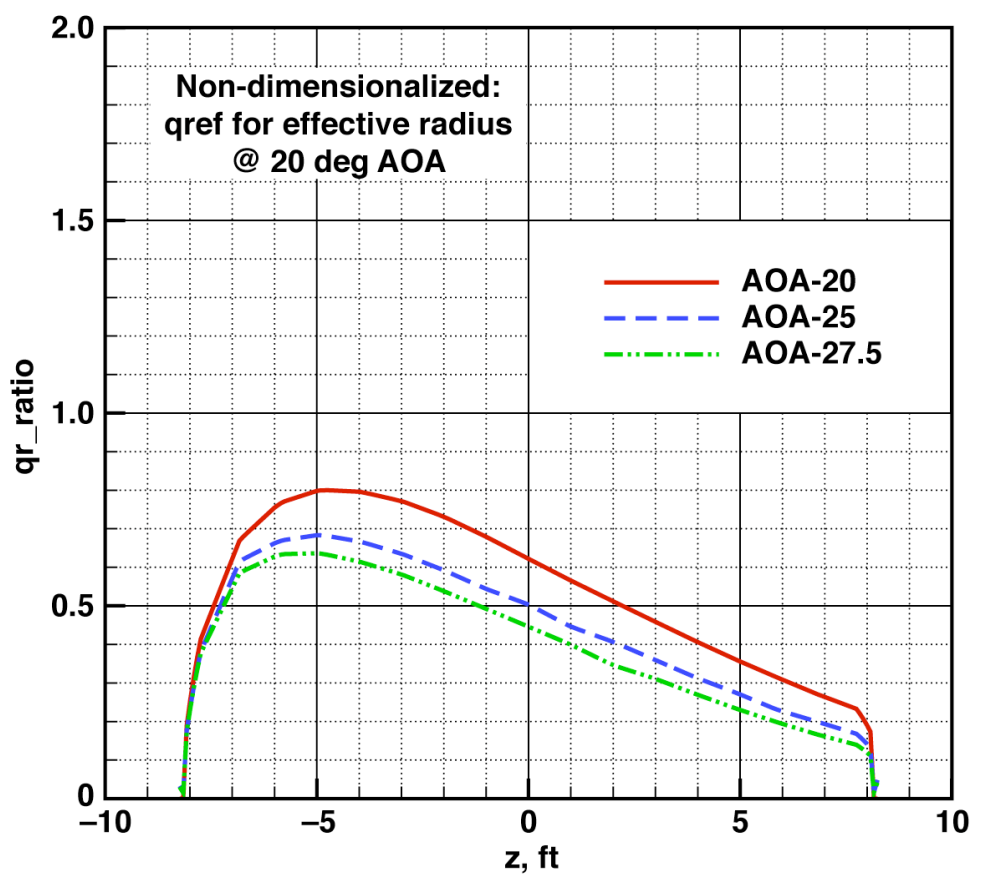

Figure 10(a). Radiative Heating - AOA Sensitivity; qref for effective radius at $20 \mathrm{deg}$ AOA. 


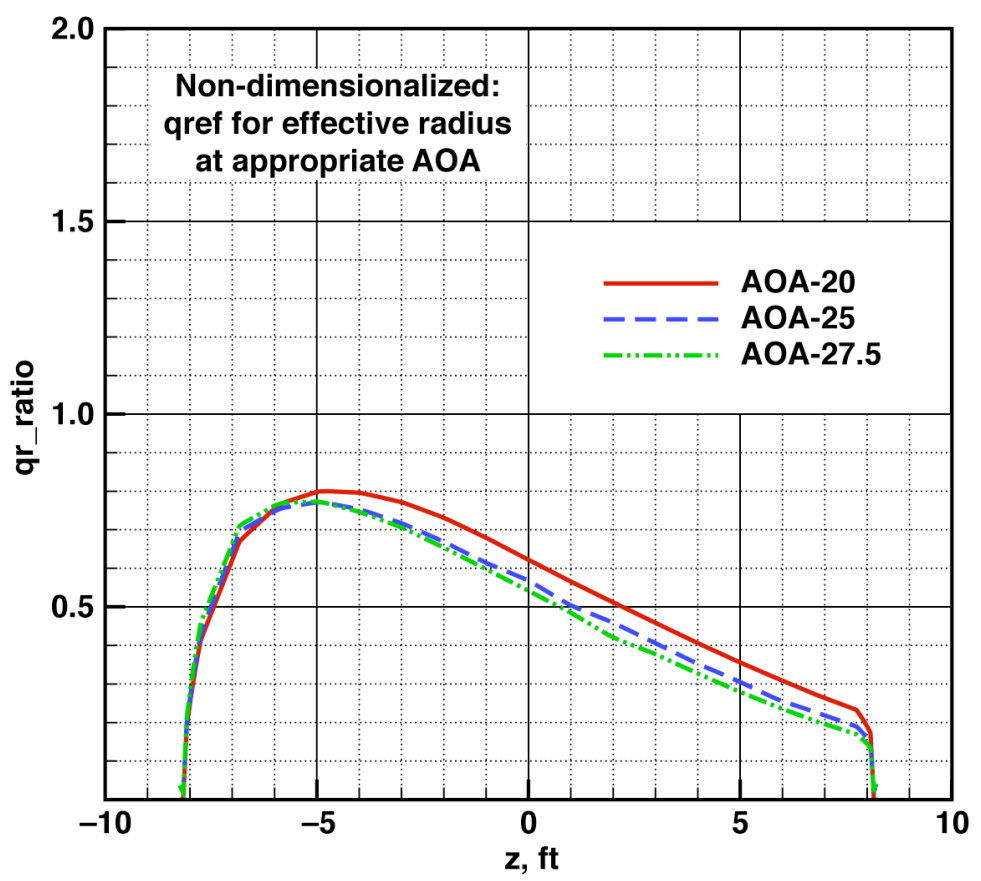

Figure 10(b). Radiative heating - AOA sensitivity; qref adjusted for effective radius at appropriate $\mathrm{AOA}$.

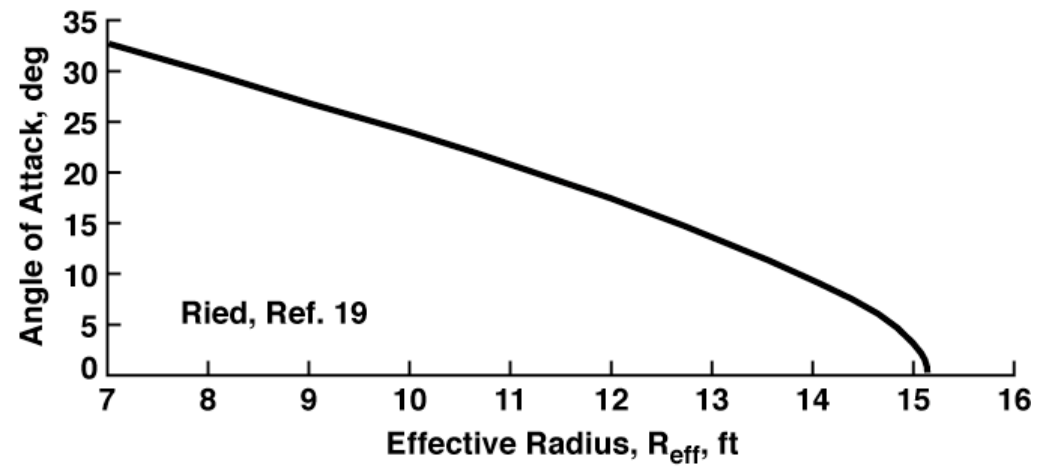

Figure 11. Effective radius for radiative heating as a function of angle of attack; Apollo Command Module 


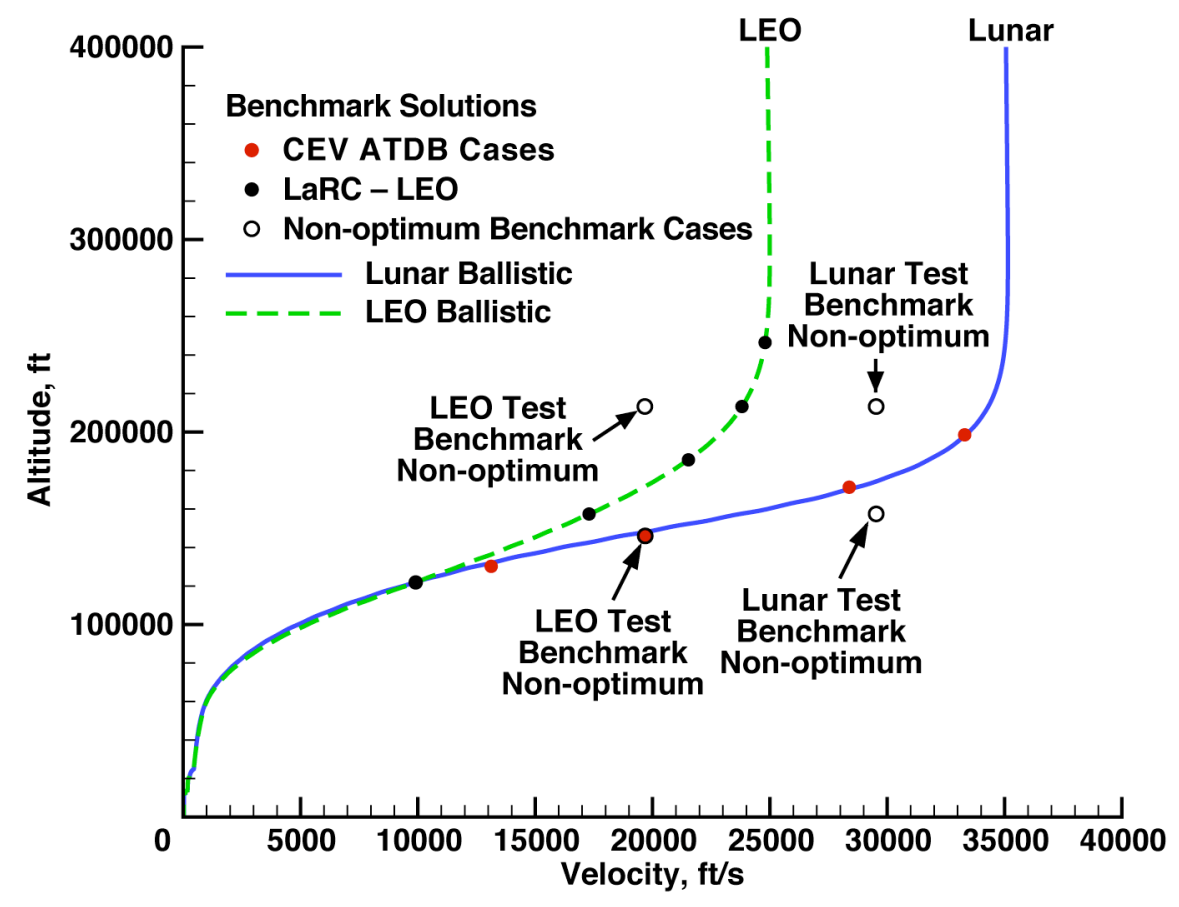

Figure 12(a). Altitude-Velocity Space - Bridging Benchmarks.

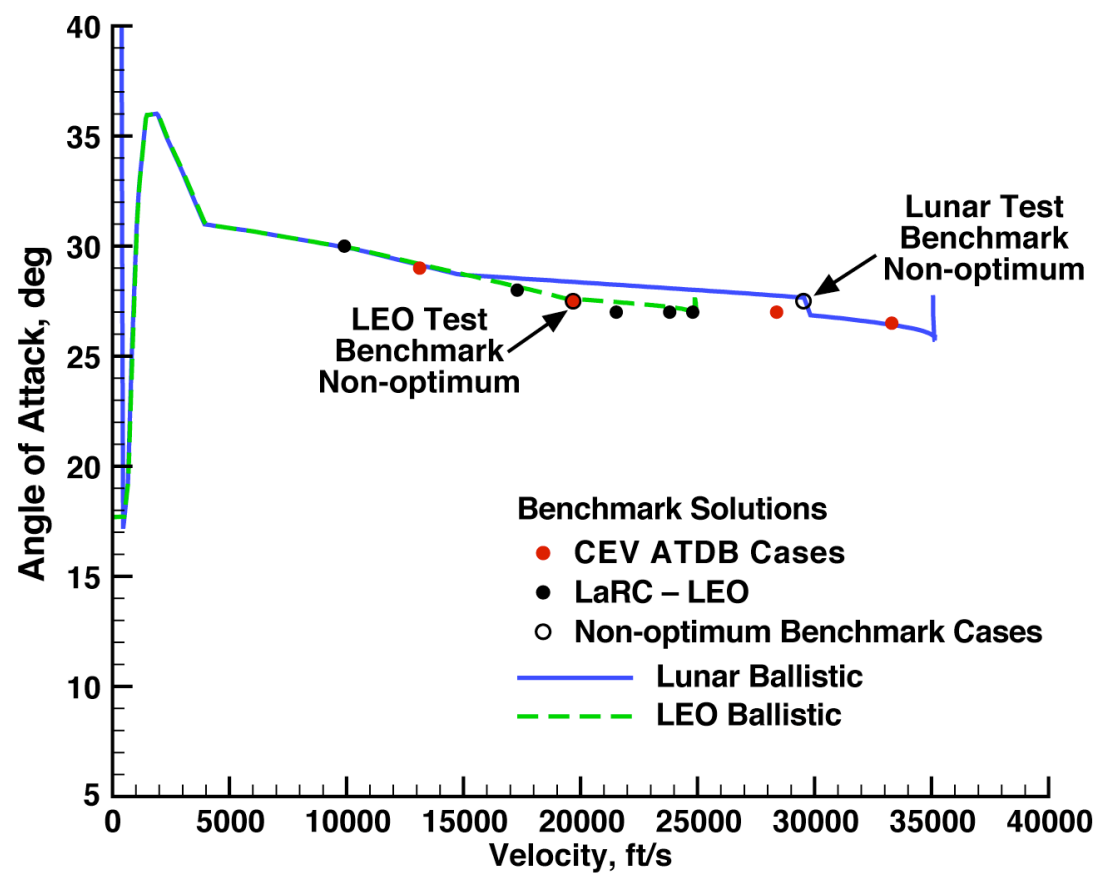

Figure 12(b). Angle of Attack-Velocity Space - Bridging Benchmarks. 


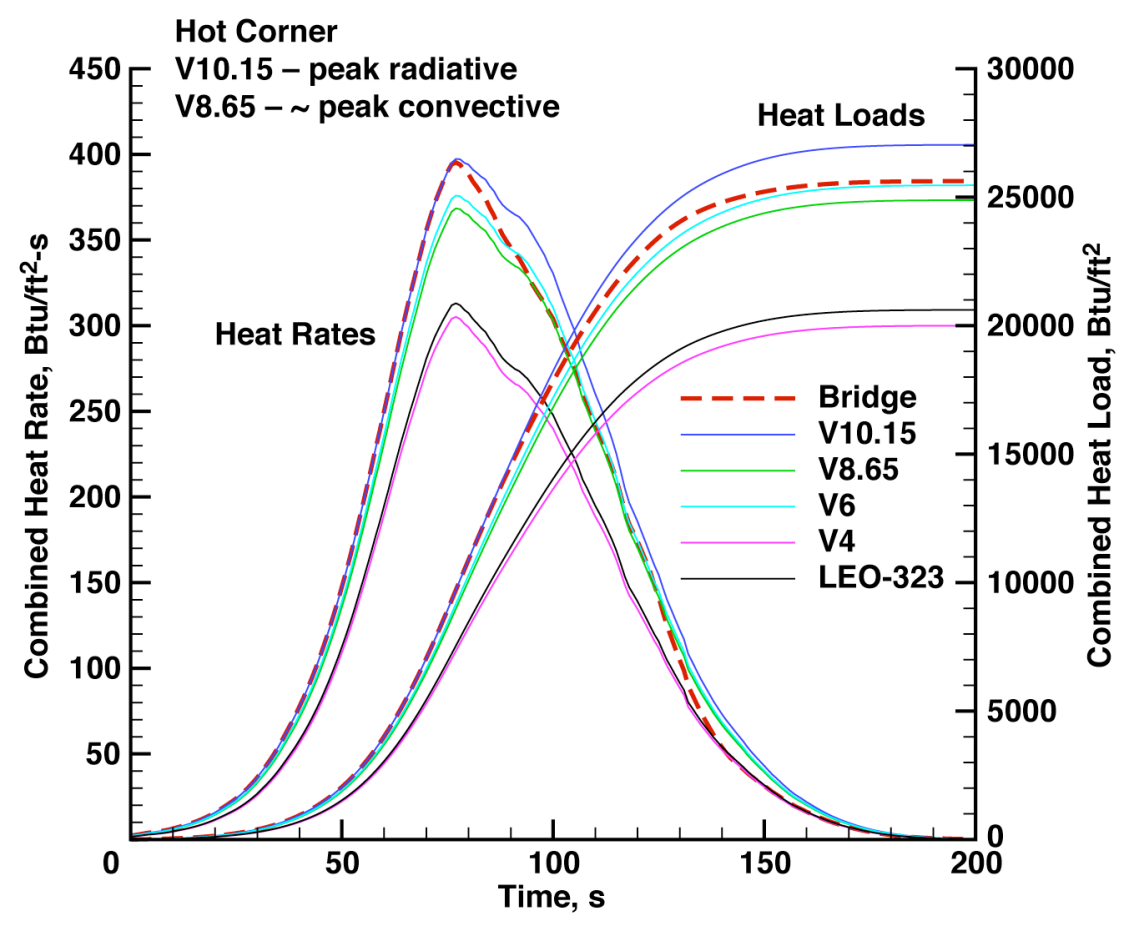

Figure 13. Single Benchmark Solutions Compared to Bridging; 28-deg Lunar Ballistic Return.

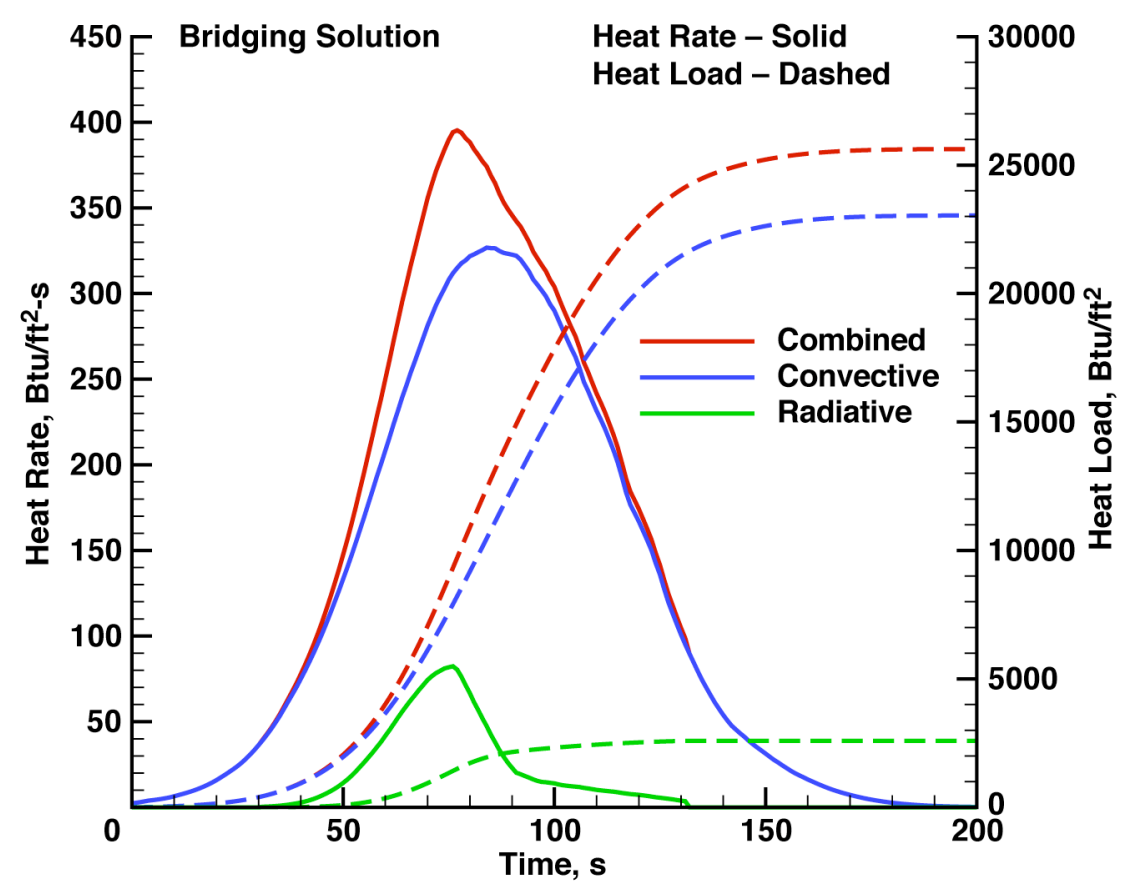

Figure 14(a). Relative Component Heating - Hot Corner; 28-deg Lunar Ballistic Return. 


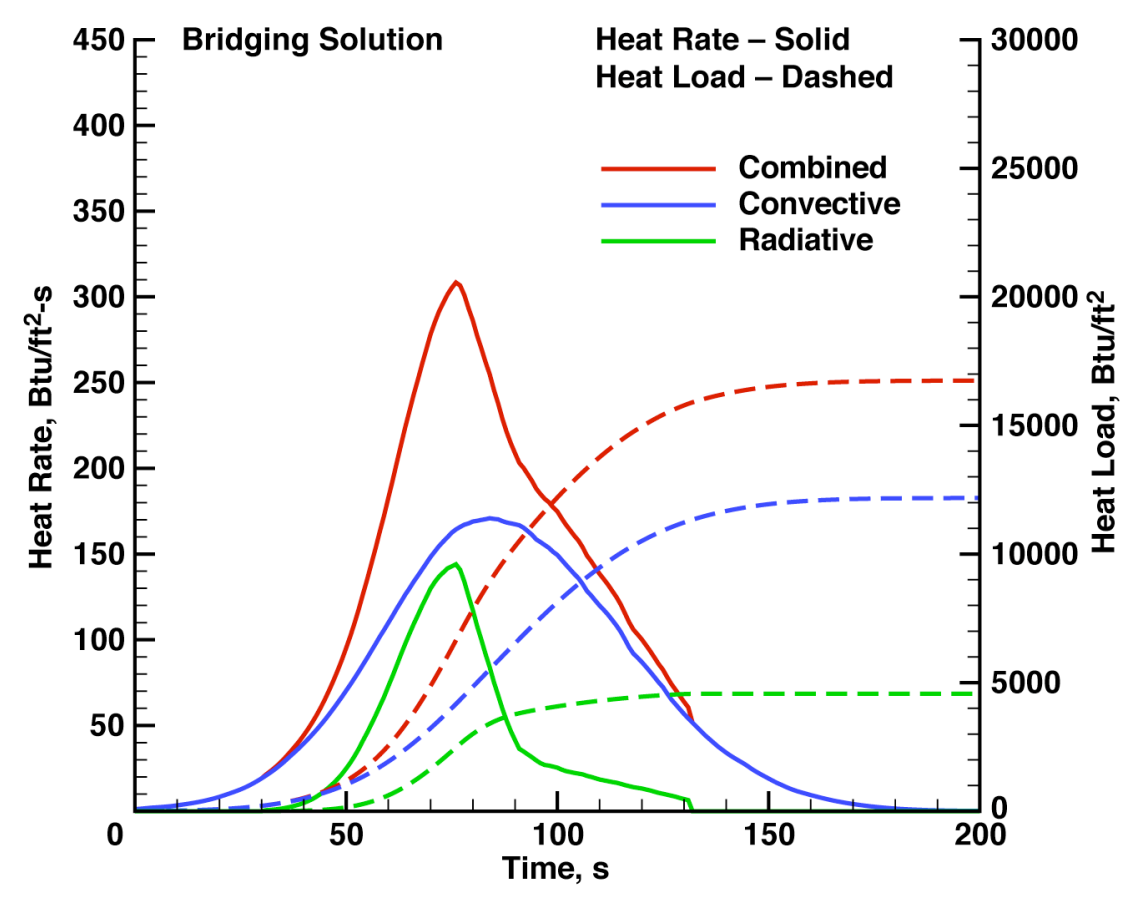

Figure 14(b). Relative Component Heating - Stagnation Point;

28-deg Lunar Ballistic Return.

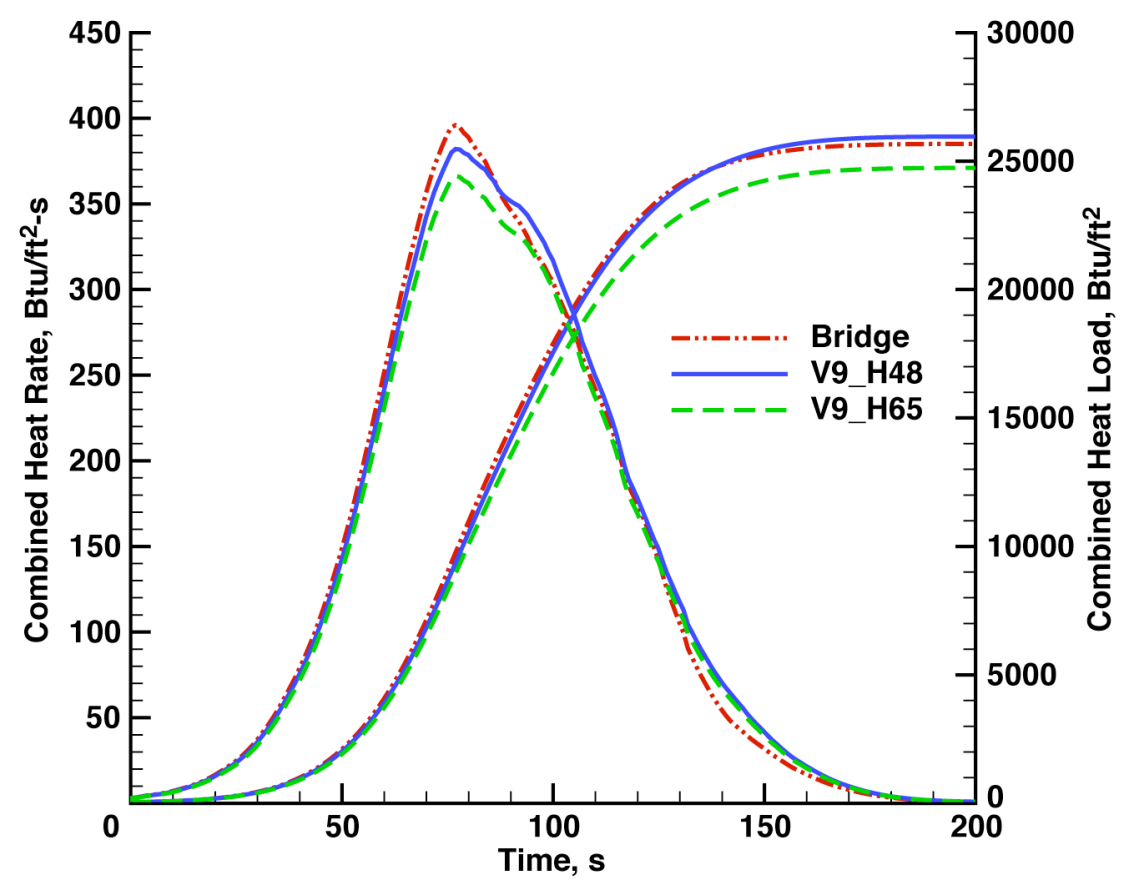

Figure 15(a). Non-optimum Benchmark Evaluation - Hot Corner;

28-deg Lunar Ballistic Return. 


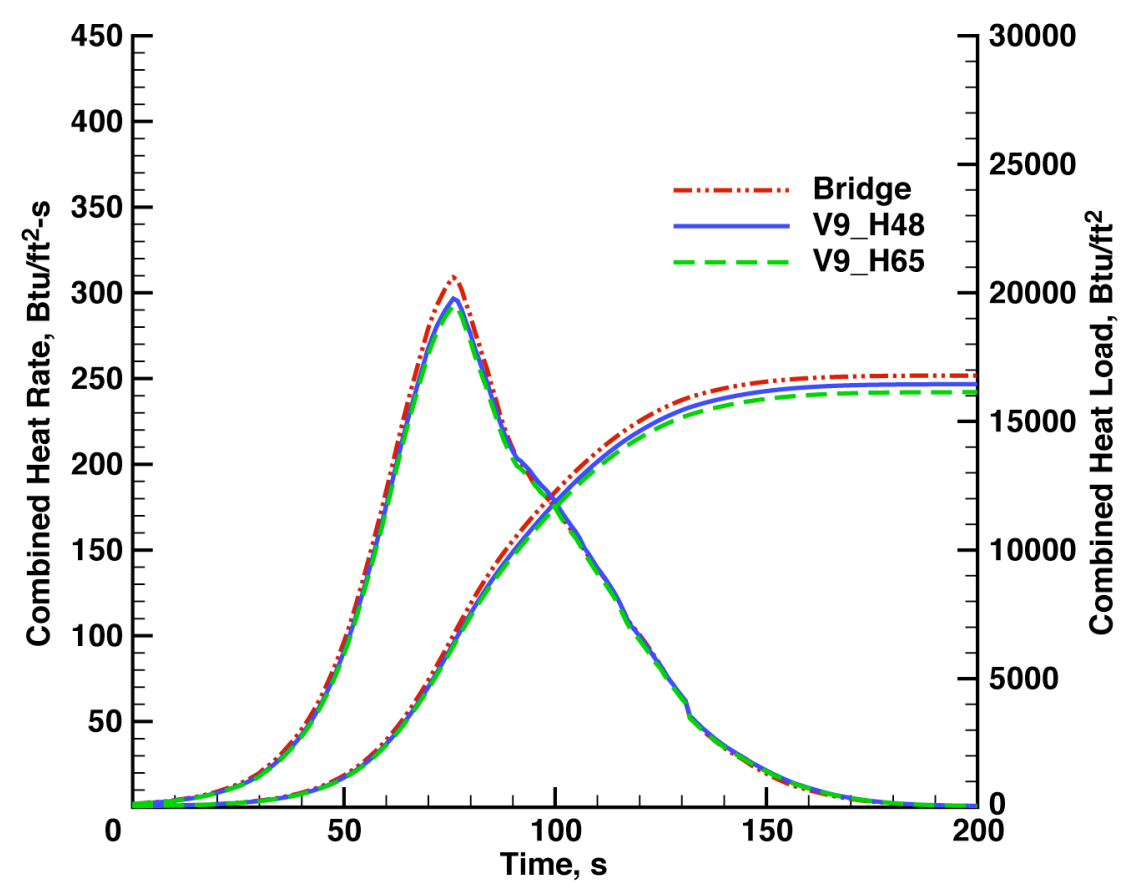

Figure 15(b). Non-optimum Benchmark Evaluation - Stagnation Point; 28-deg Lunar Ballistic Return.

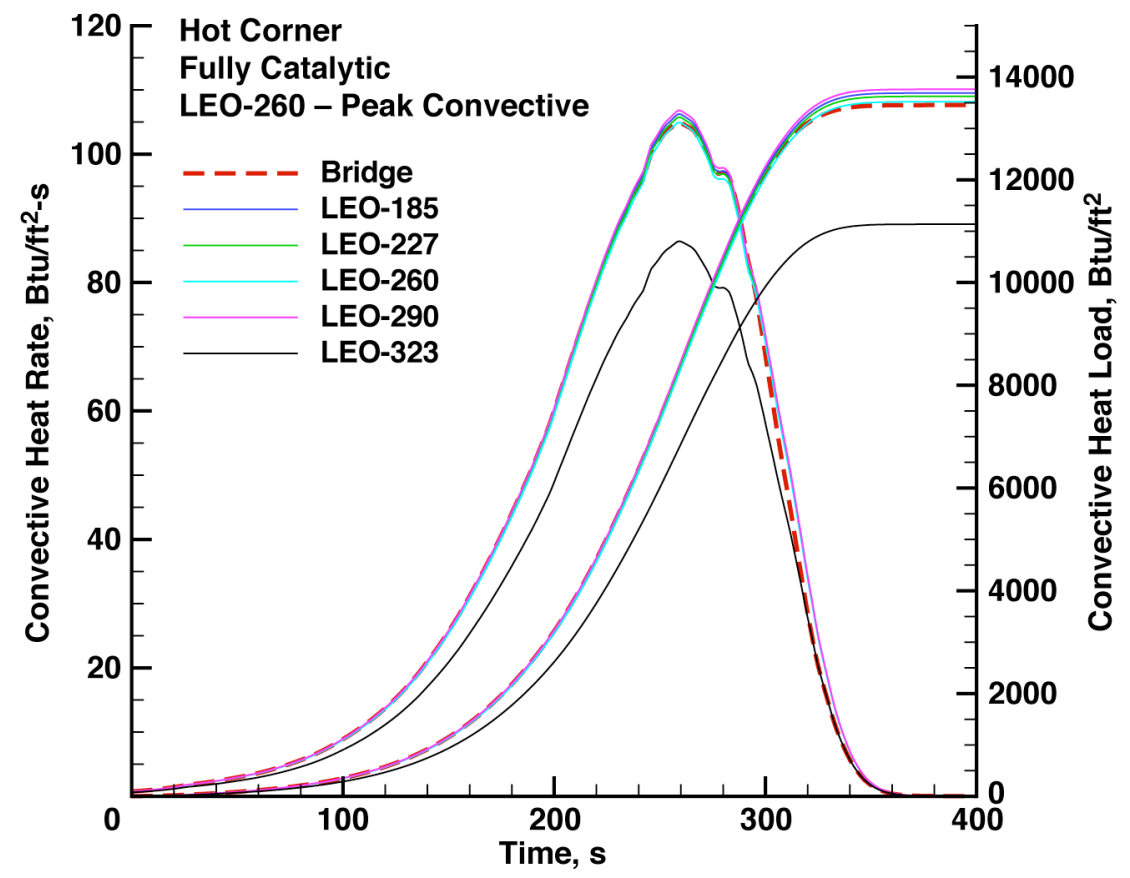

Figure 16(a). Single Benchmark Solutions Compared to Bridging - Hot Corner; 28-deg LEO Ballistic Return. 


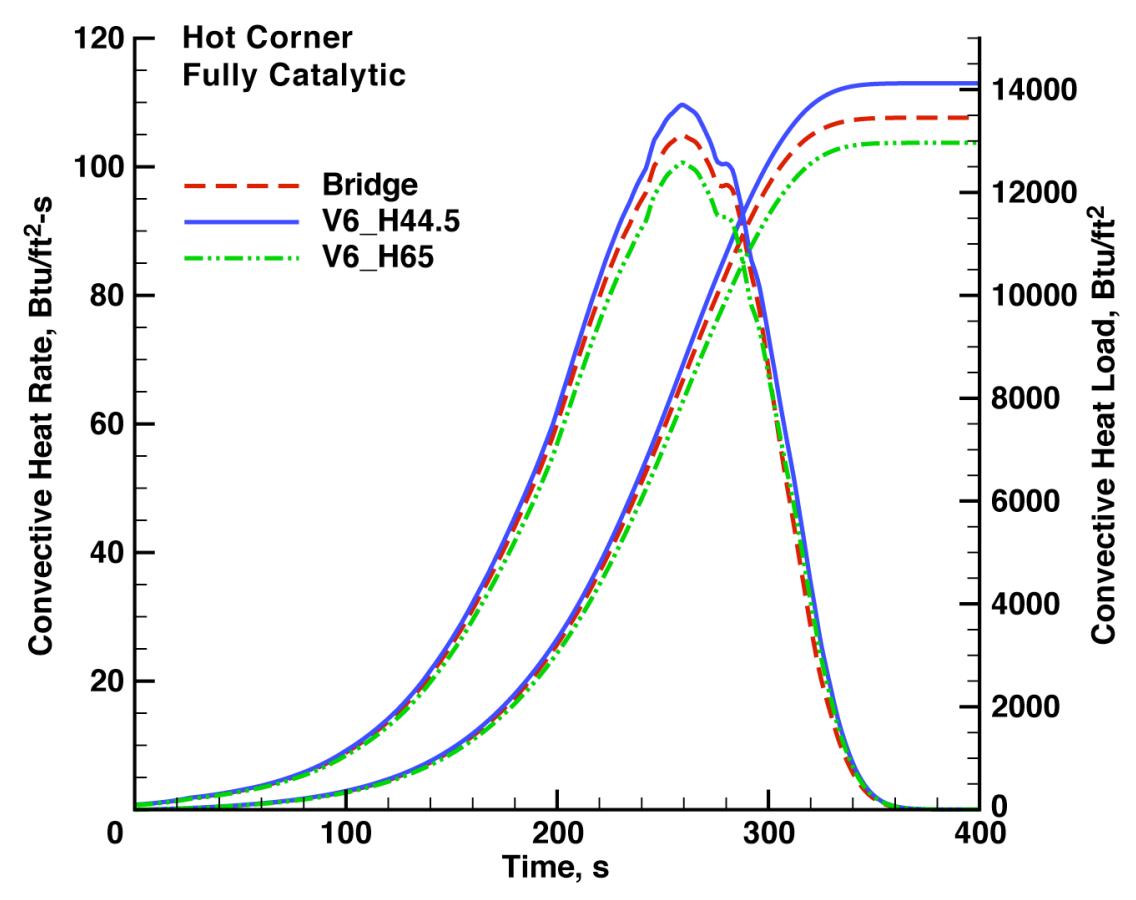

Figure 16(b). Non-optimum Benchmark Evaluation - Hot Corner; 28-deg LEO Ballistic Return.

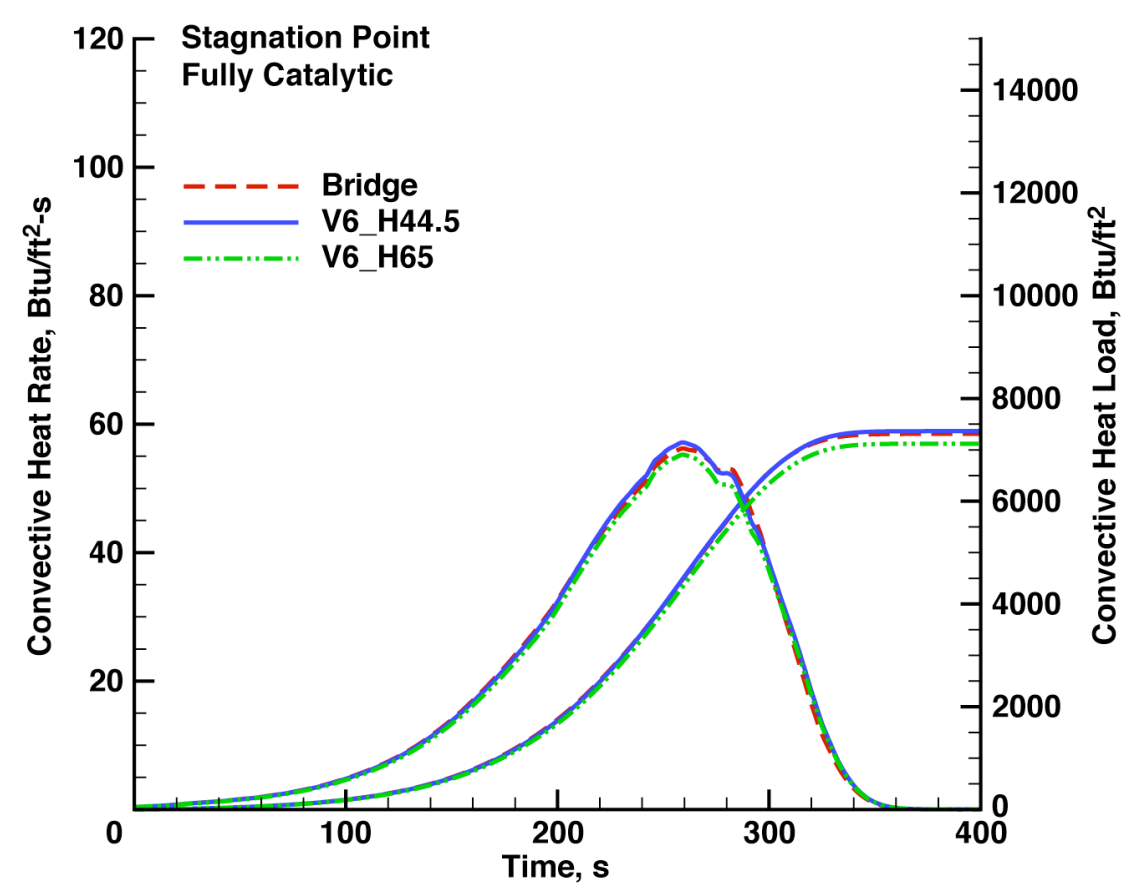

Figure 16(c). Non-optimum Benchmark Evaluation - Stagnation Point; 28-deg LEO Ballistic Return. 


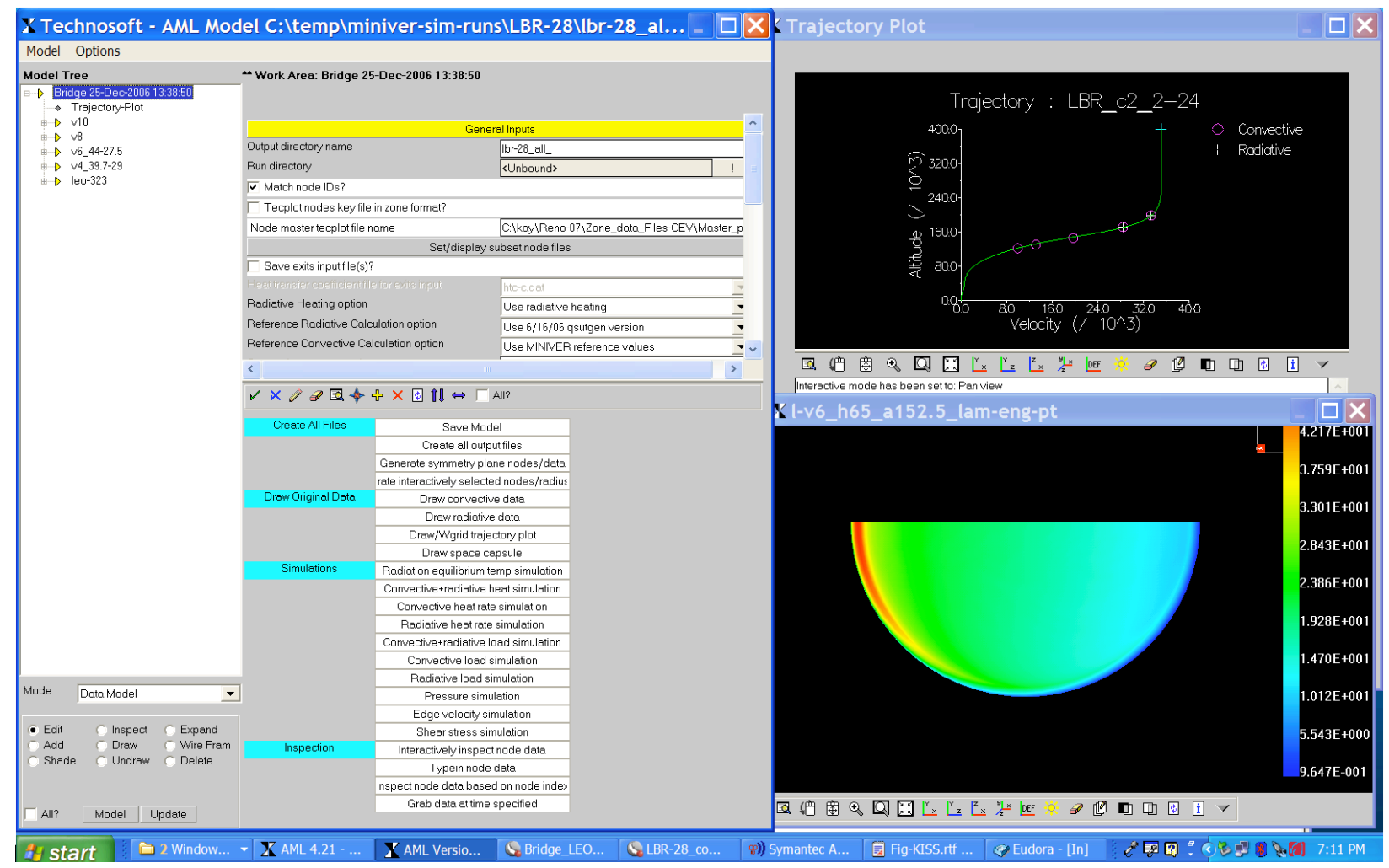

Figure 17(a). KISS Graphical Interface Display.

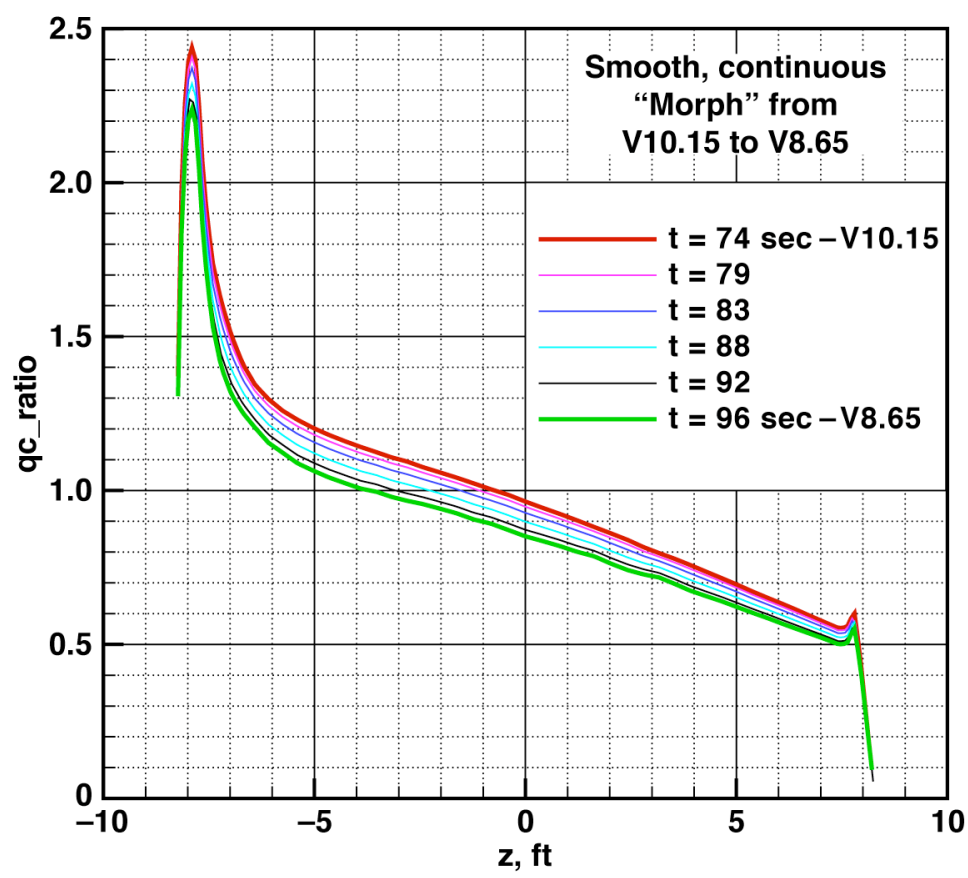

Figure 17(b). "Morphing" verification - convective; 28-deg Lunar Ballistic Return. 


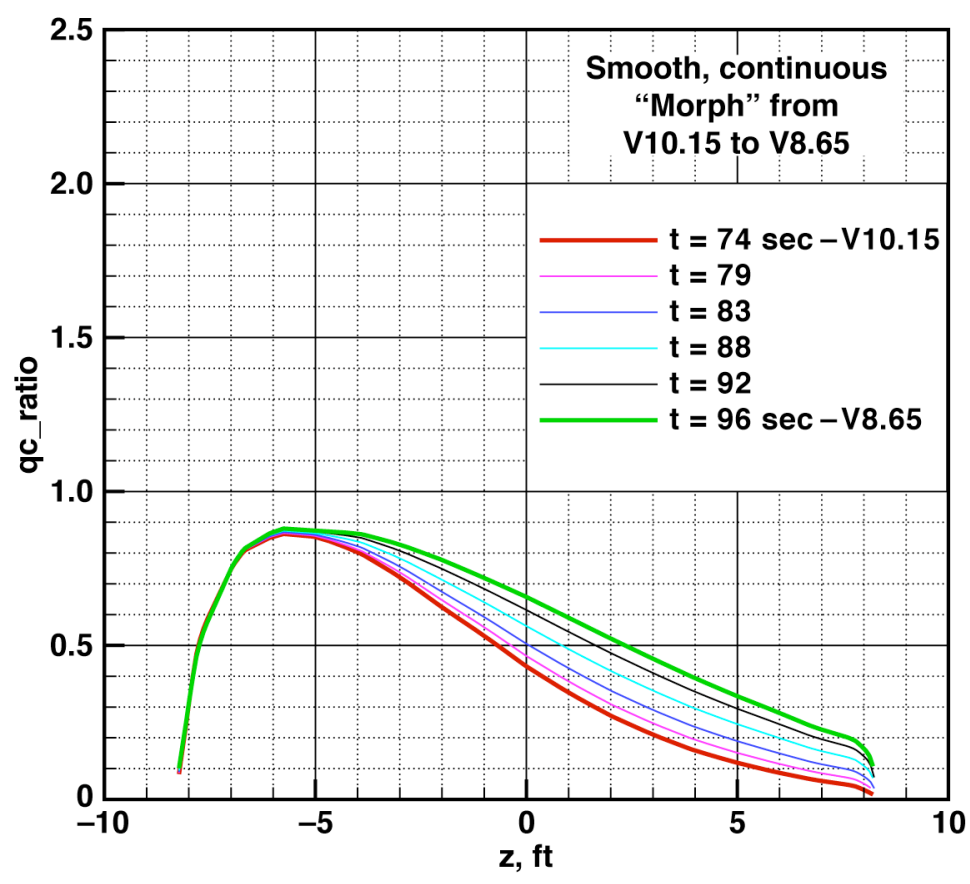

Figure 17(c). "Morphing" Verification - Radiative;

28-deg Lunar Ballistic Return.

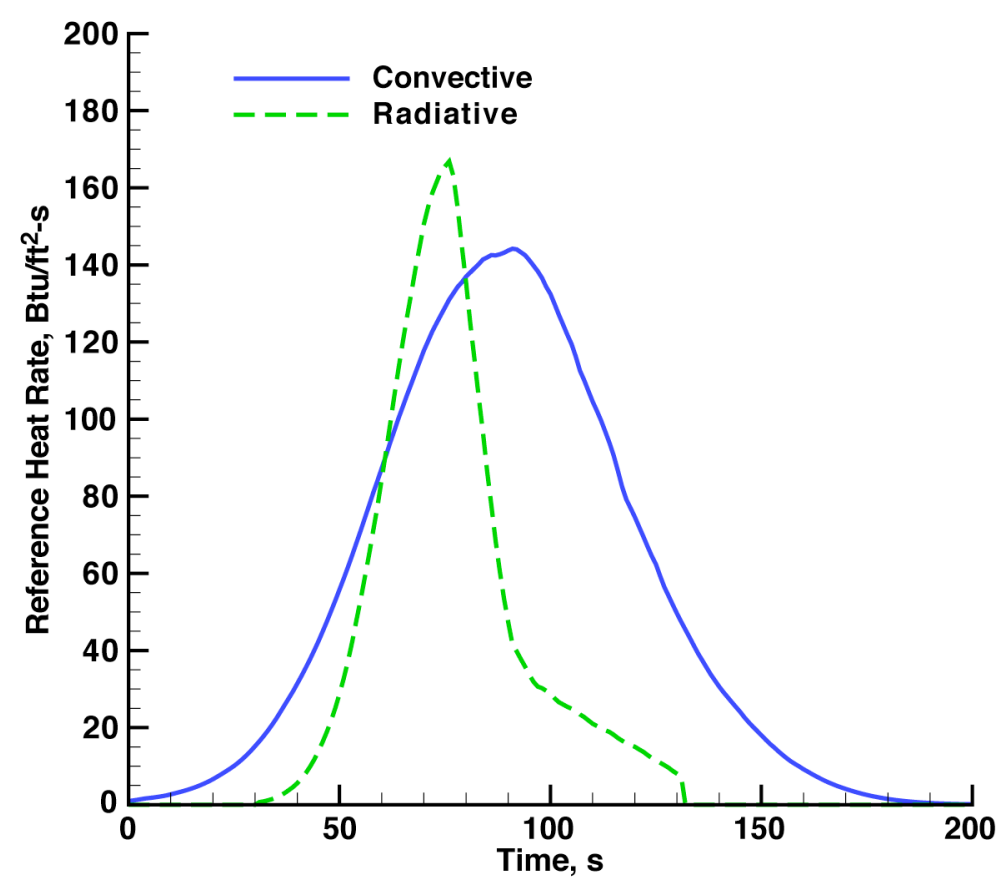

Figure 18. Reference Heating Rate Histories for "Morphing" Verification Test; 28-deg Lunar Ballistic Return. 


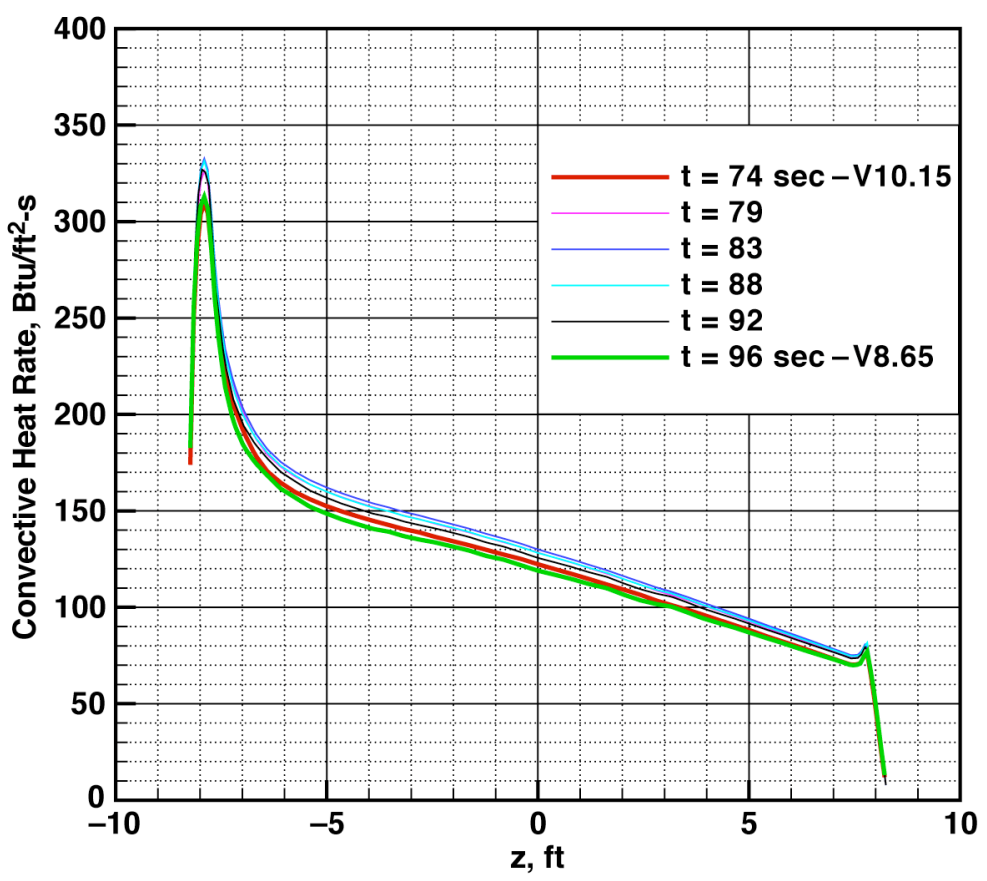

Figure 19(a). Convective Heating Rate from "Morphing" Verification Test; 28-deg Lunar Ballistic Return.

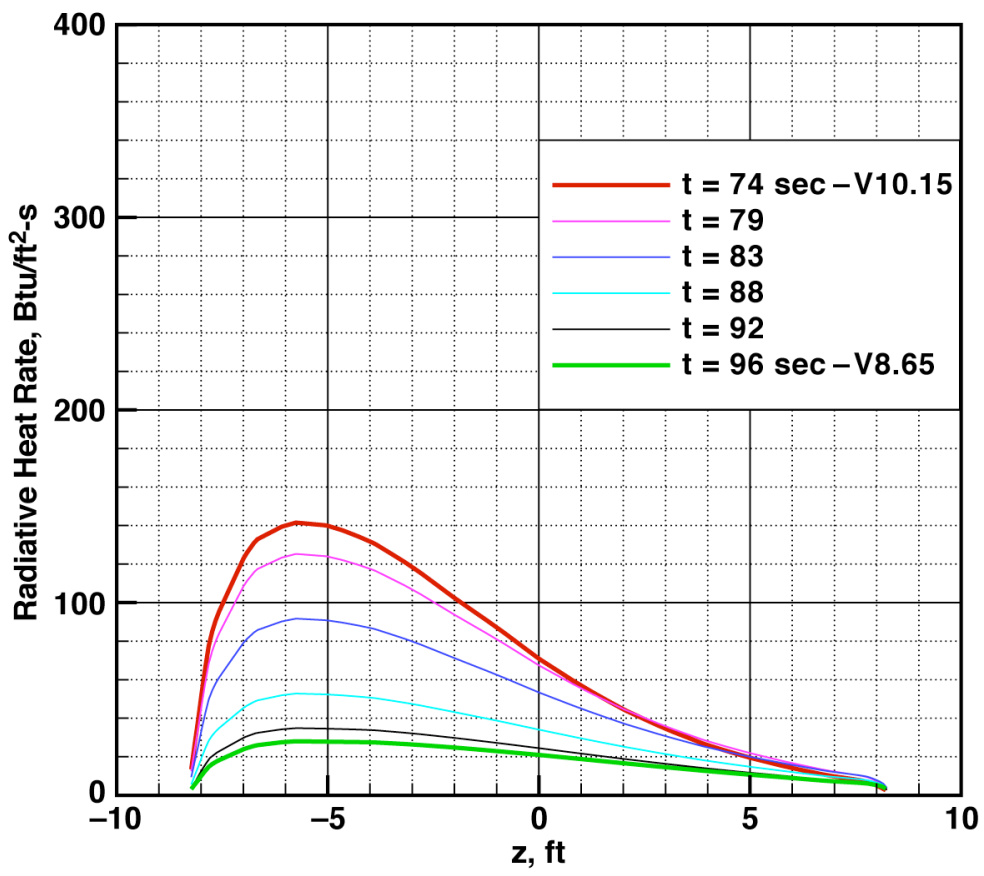

Figure 19(b). Radiative Heating Rate from "Morphing" Verification Test; 28-deg Lunar Ballistic Return. 


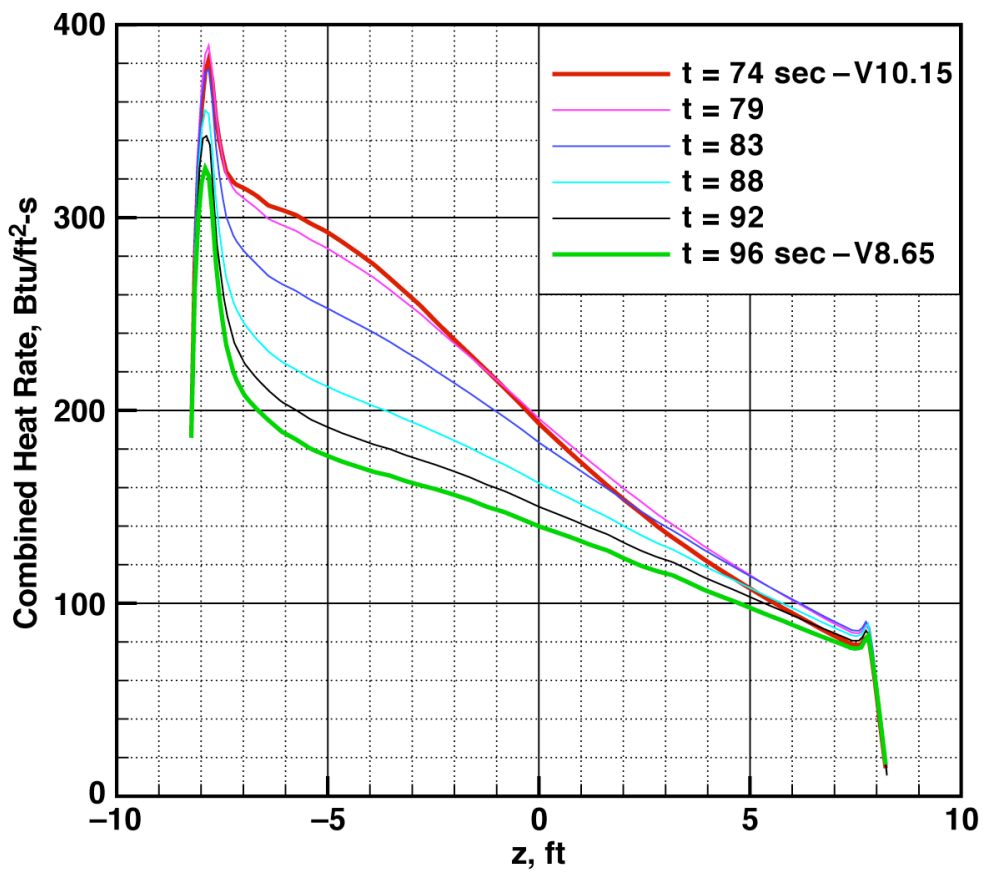

Figure 19(c). Combined Heating Rate from "Morphing" Verification Test; 28-deg Lunar Ballistic Return. 


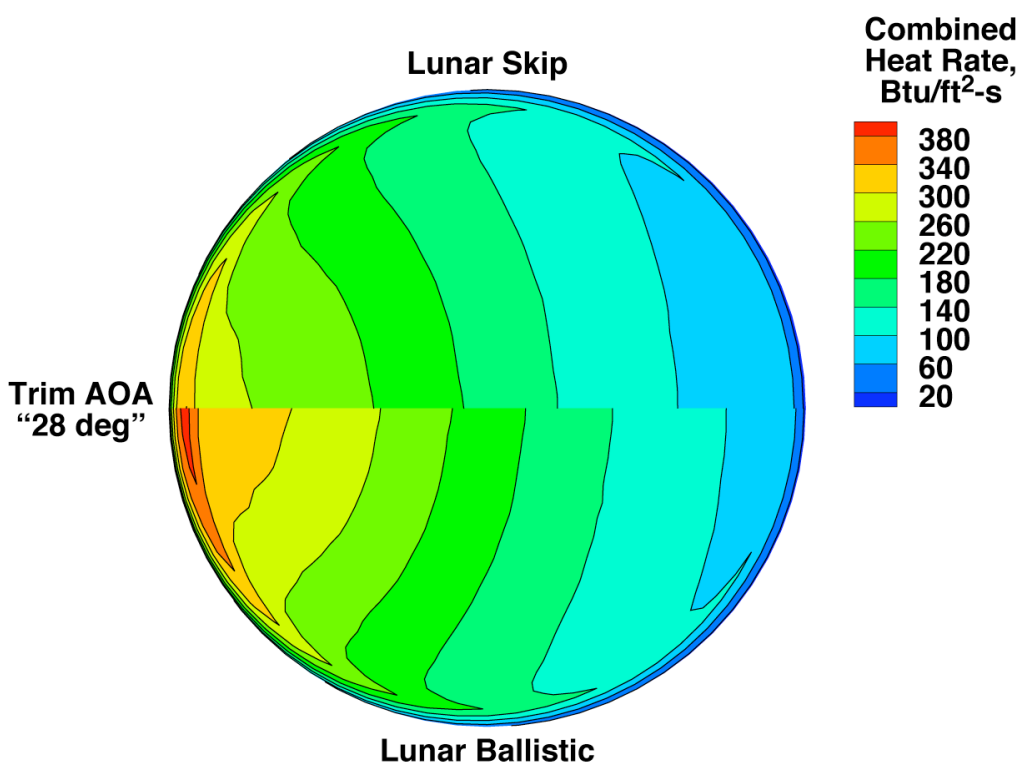

Figure 20(a). Maximum Heat Rate Comparison - Lunar Return; Skip Versus Ballistic.

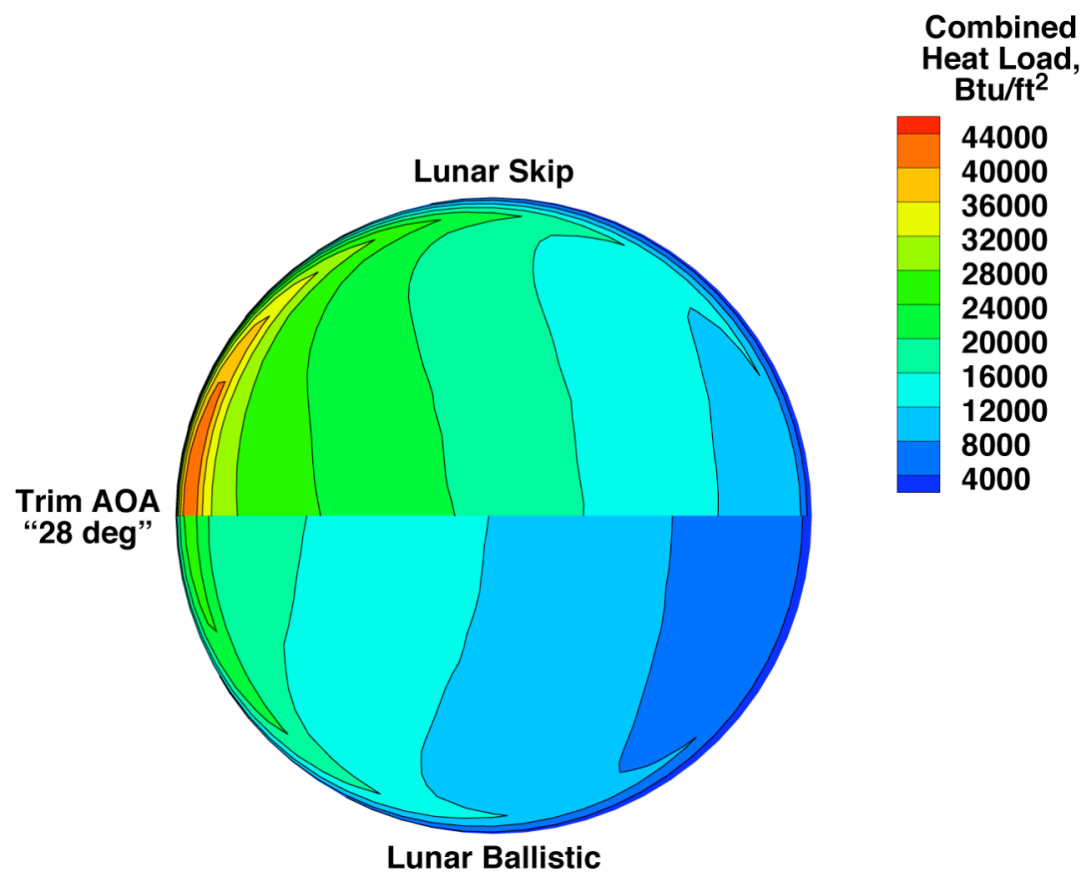

Figure 20(b). Integrated Heat Load Comparison - Lunar Return; Skip Versus Ballistic. 


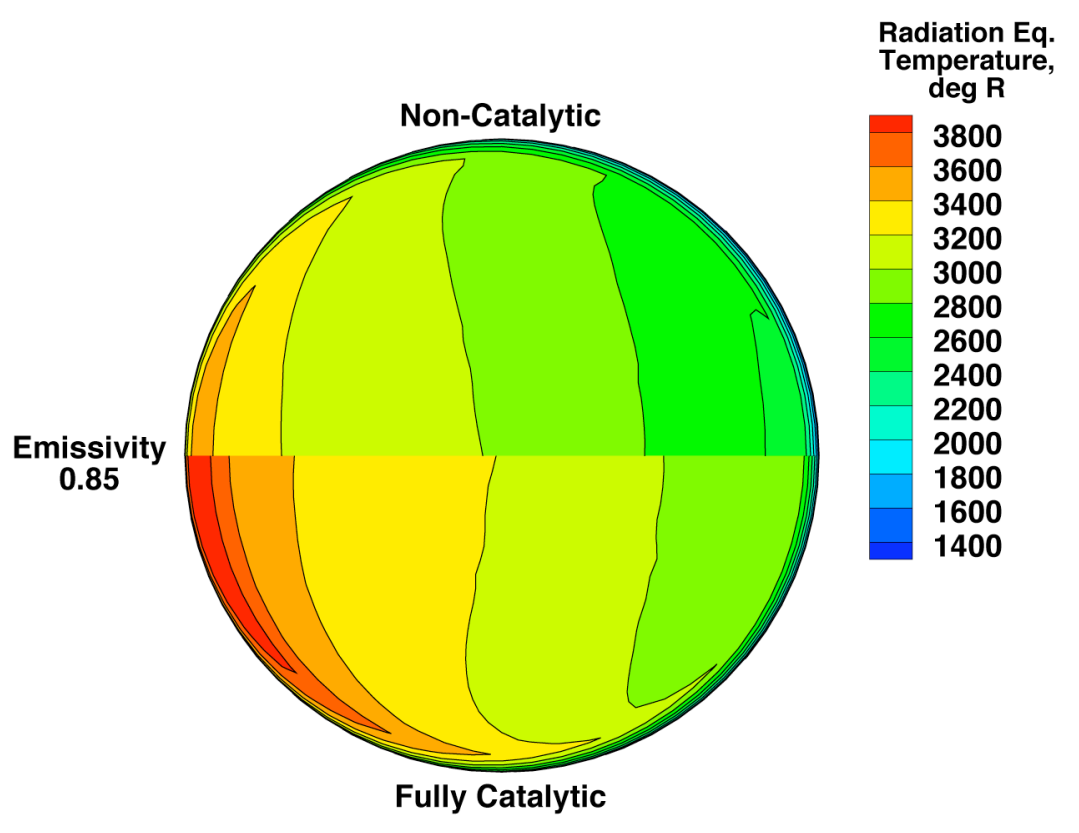

Figure 21(a). Maximum Radiation Equilibrium Temperature - LEO Ballistic Return; Fully Catalytic Versus Non-catalytic.

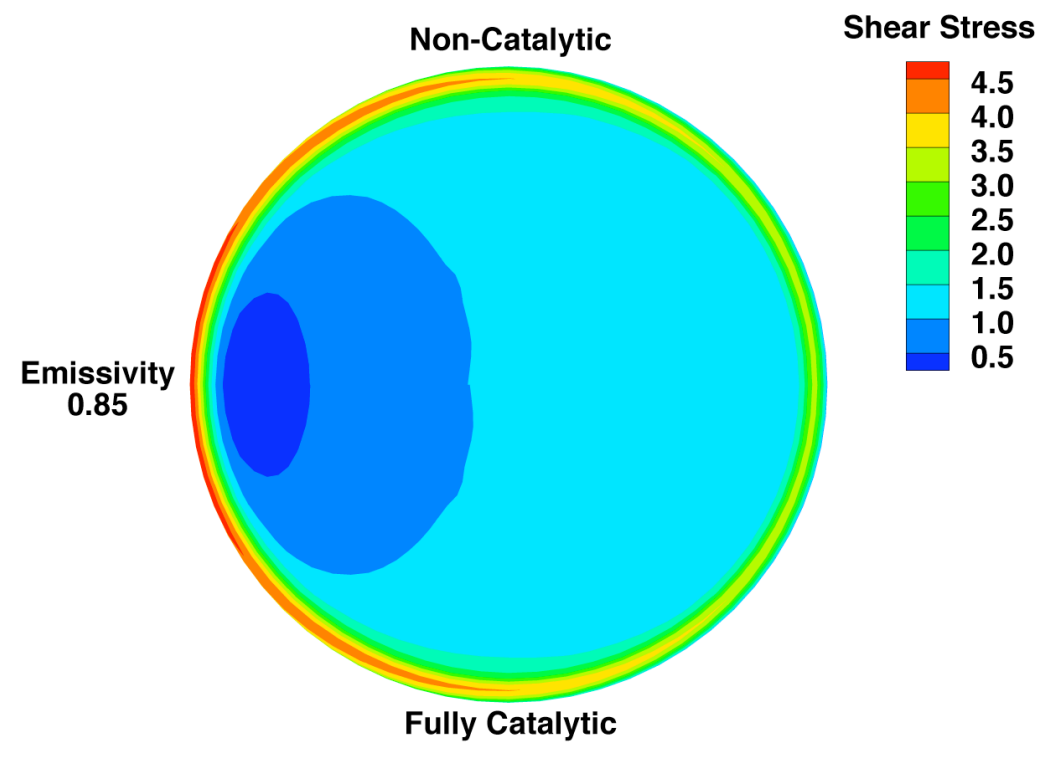

Figure 21(b). Shear Stress Distribution. 


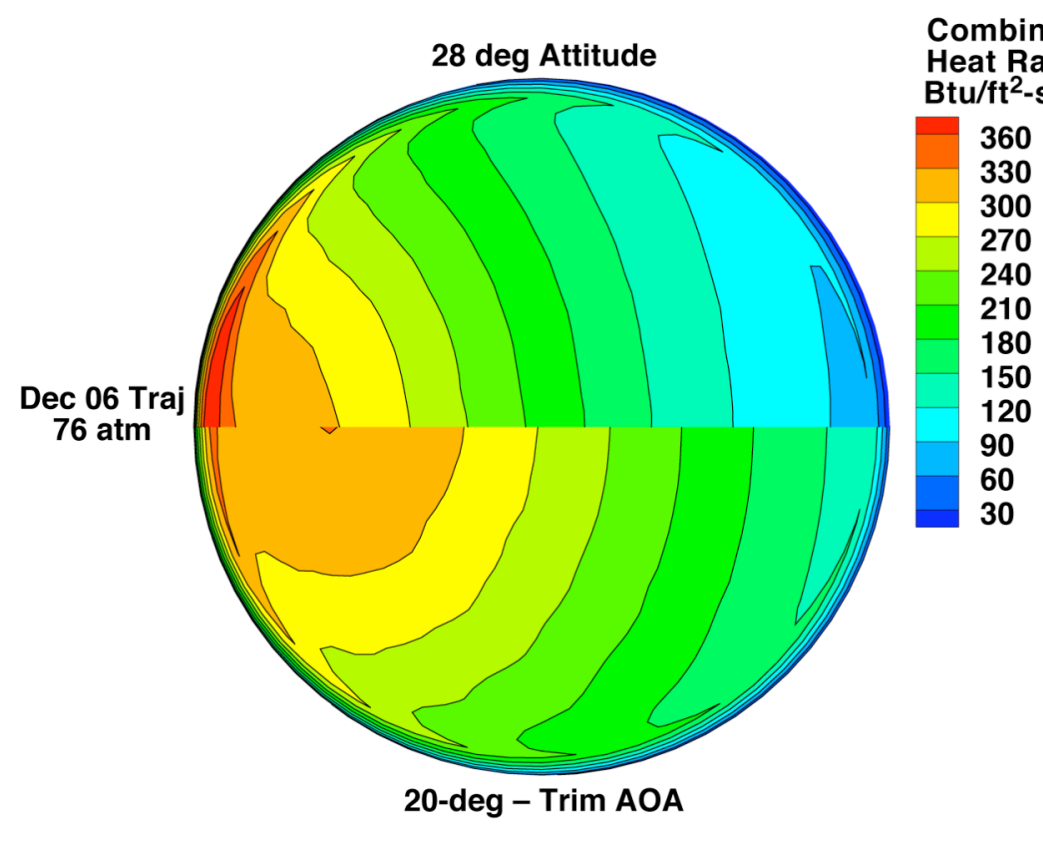

Figure 22(a). Maximum Heat Rate Comparison - Lunar Ballistic; “28-deg” Versus 20-deg Trim AOA.

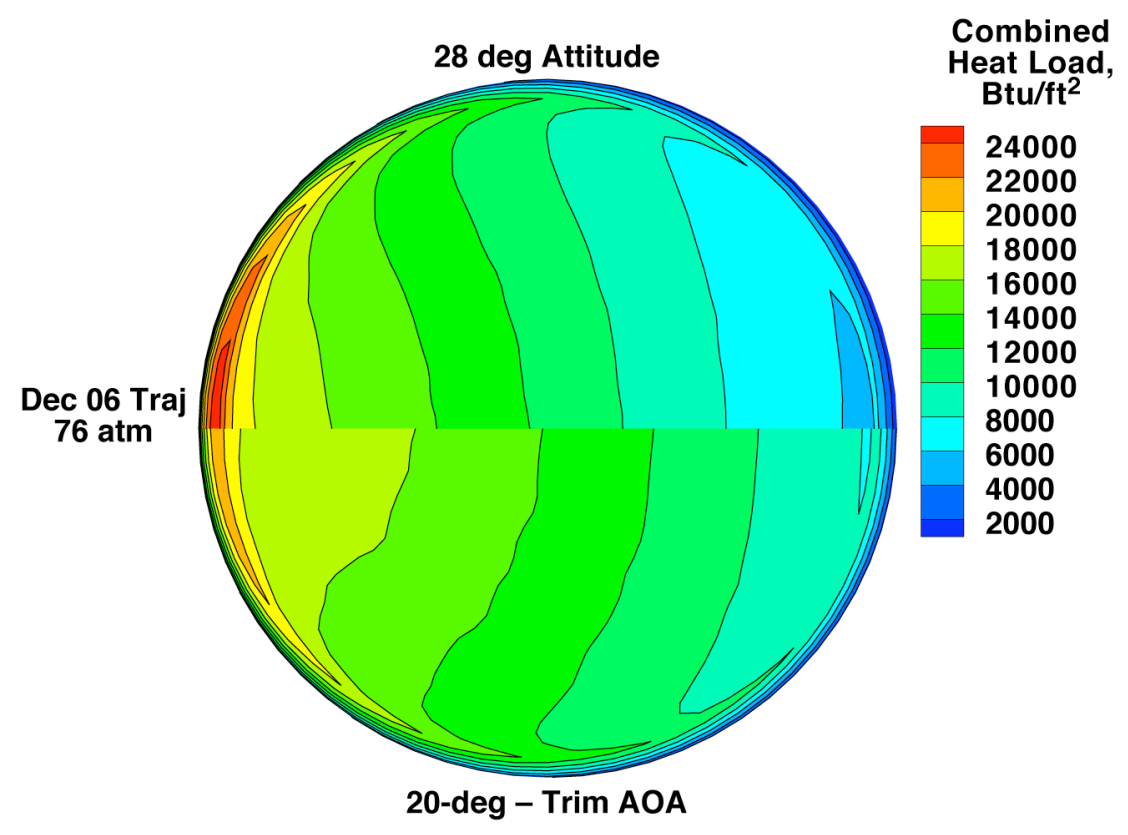

Figure 22(b). Integrated Heat Load Comparison - Lunar Ballistic; “28-deg” Versus 20-deg Trim AOA. 


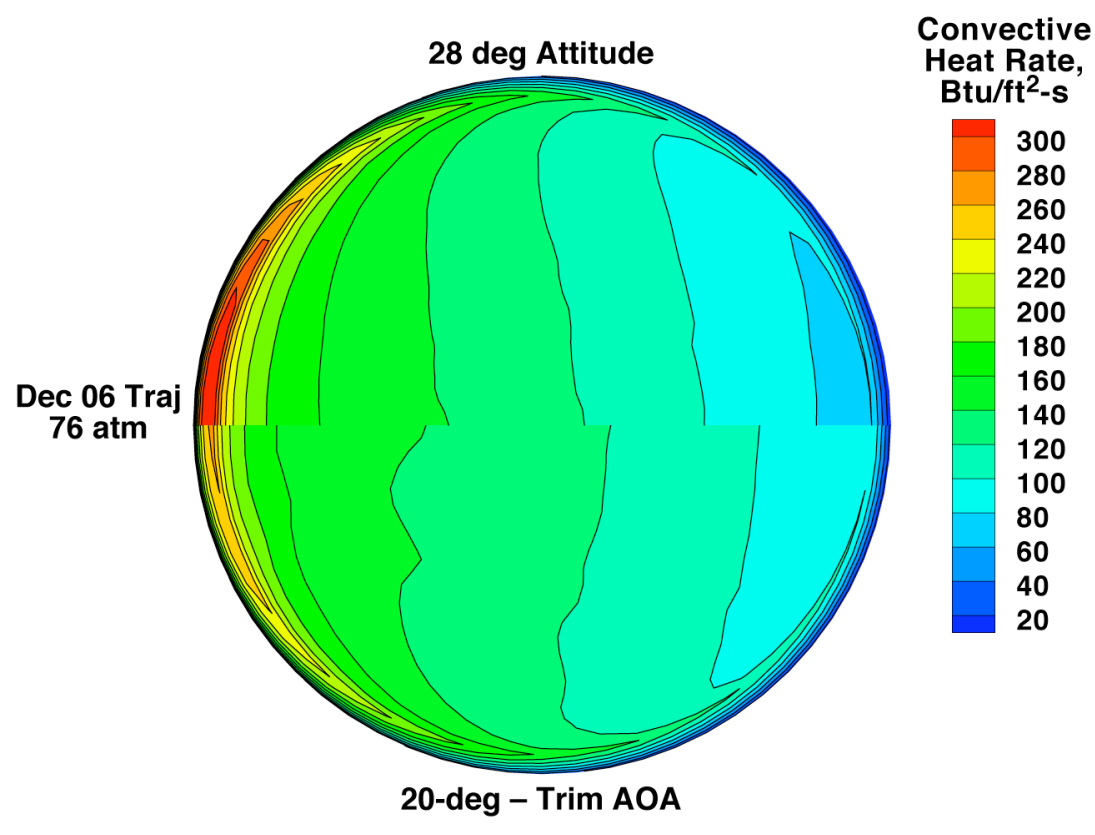

Figure 22(c). Maximum Convective Heat Rate Comparison - Lunar Ballistic; “28-deg" Versus 20-deg Trim AOA.

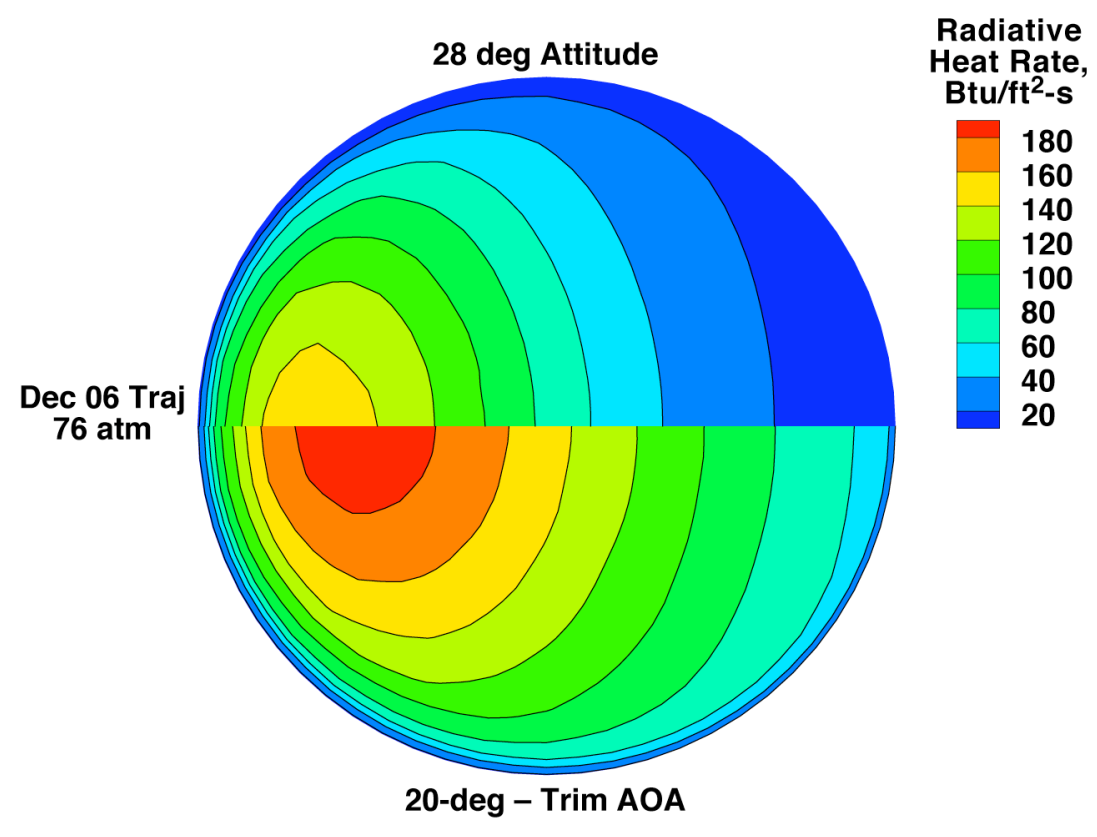

Figure 22(d). Maximum Radiative Heat Rate Comparison - Lunar Ballistic; “28-deg" Versus 20-deg Trim AOA. 


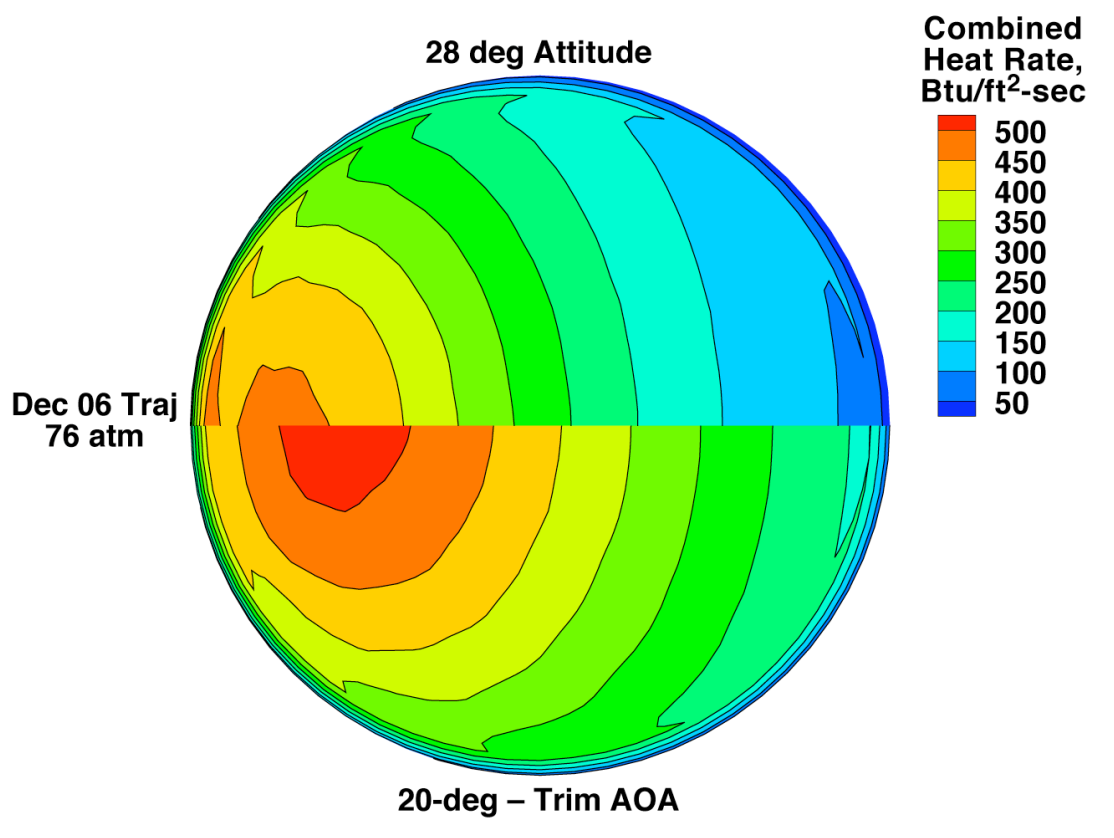

Figure 23(a). Maximum Heat Rate Comparison - Lunar Ballistic; Relative Influence - 28 vs 20 deg - Double Radiation.

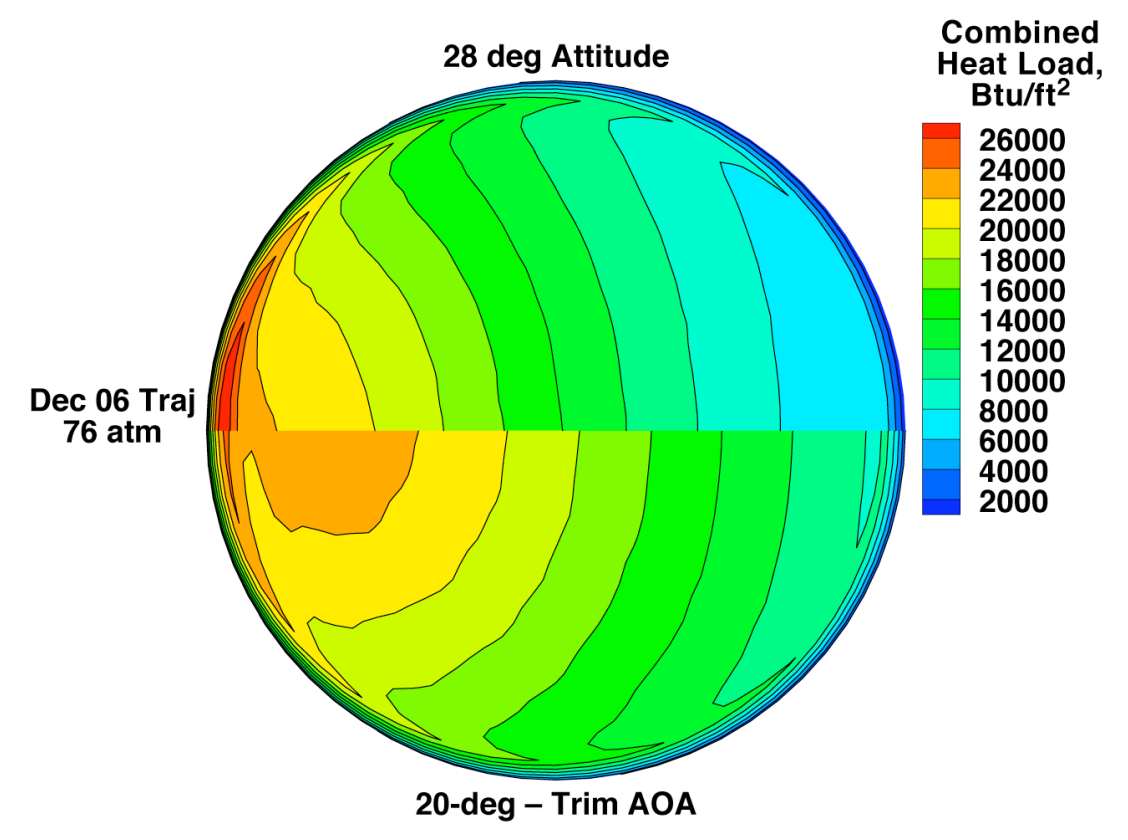

Figure 23(b). Integrated Heat Load Comparison - Lunar Ballistic; Relative Influence - 28 vs 20 deg - Double Radiation. 


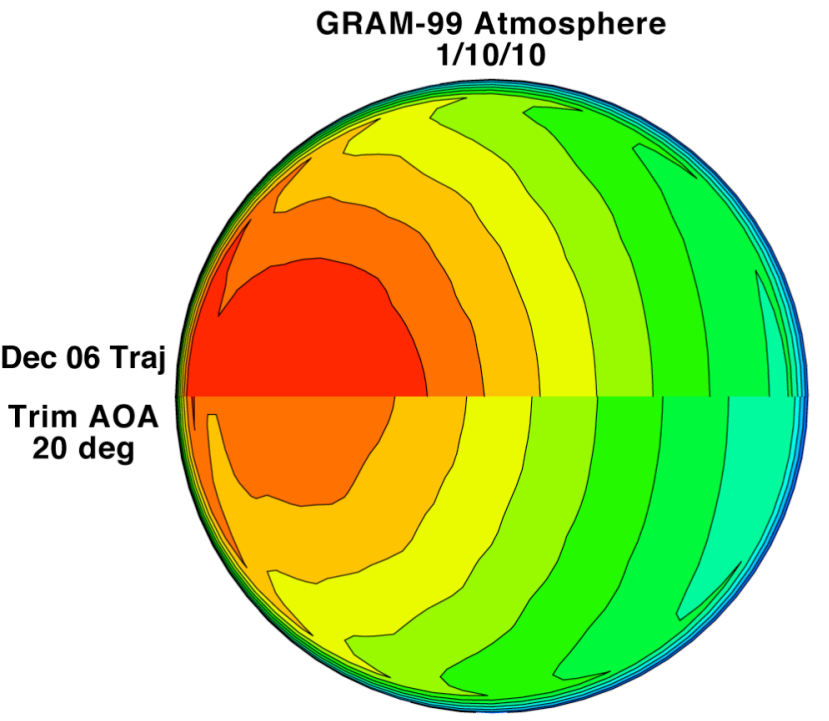

Combined

Heat Rate,

Btu/ft ${ }^{2}-\mathrm{s}$

340

310

280

250

220

190

160

130

100

70

40

76 Standard Atmosphere

Figure 24(a). Heat Rate Comparisons - Lunar Ballistic Return; 76 Atmosphere Versus GRAM-99 1/1/10.

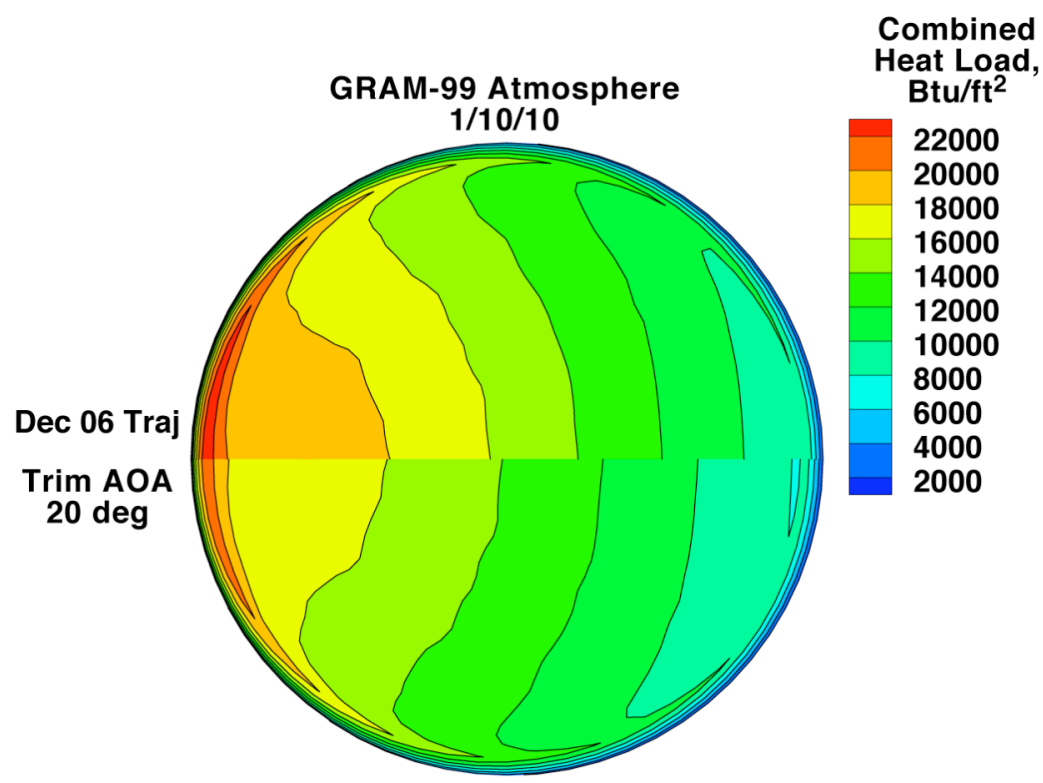

76 Standard Atmosphere

Figure 24(b). Integrated Heat Load Comparisons - Lunar Ballistic Return; 76 Atmosphere Versus GRAM-99 1/1/10. 


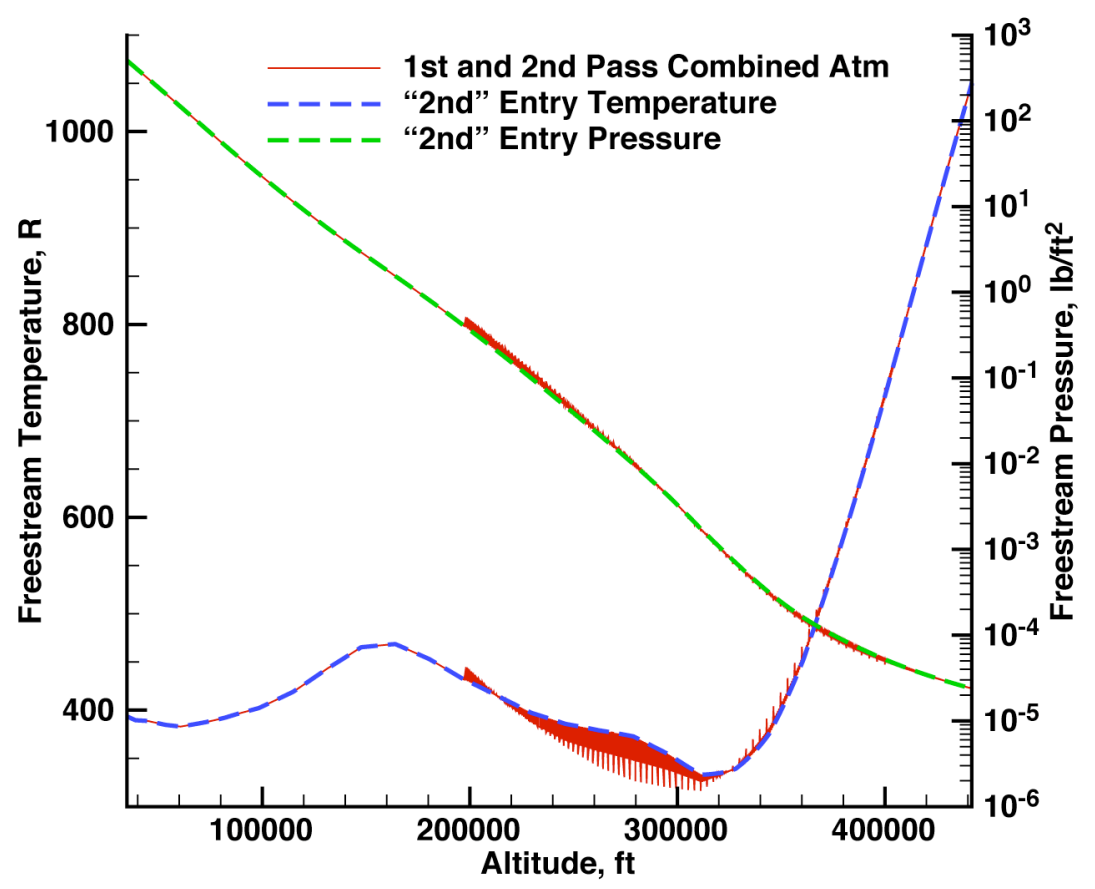

Figure 25. GRAM-99 Atmosphere for 1/10/10 Utah Test Range; Lunar Skip Entry. 